\title{
Genome-wide CRISPR activation screen identifies candidate receptors for SARS-CoV-2 entry
}

\author{
Shiyou $\mathrm{Zhu}^{1 \dagger}$, Ying Liu ${ }^{1 \dagger}$, Zhuo Zhou ${ }^{1 \dagger}$, Zhiying Zhang ${ }^{2 \dagger}$, Xia Xiao ${ }^{3}$, Zhiheng Liu ${ }^{1}$, \\ Ang Chen ${ }^{1,4}$, Xiaojing Dong ${ }^{3}$, Feng Tian ${ }^{1}$, Shihua Chen ${ }^{2,4}$, Yiyuan $\mathrm{Xu}^{1}$, Chunhui Wang ${ }^{1}$, \\ Qiheng $\mathrm{Li}^{1}$, Xuran Niu ${ }^{1}$, Qian Pan ${ }^{1}$, Shuo Du ${ }^{2}$, Junyu Xiao ${ }^{2 *}$, Jianwei $\mathrm{Wang}^{3^{*}}$ \& \\ Wensheng $\mathrm{Wei}^{{ }^{*}}$

\begin{abstract}
${ }^{1}$ Biomedical Pioneering Innovation Center, Beijing Advanced Innovation Center for Genomics, Peking-Tsinghua Center for Life Sciences, Peking University Genome Editing Research Center, State Key Laboratory of Protein and Plant Gene Research, School of Life Sciences, Peking University, Beijing 100871, China;

${ }^{2}$ State Key Laboratory of Protein and Plant Gene Research, School of Life Sciences, Peking University; Peking-Tsinghua Center for Life Sciences, Peking University; Beijing Advanced Innovation Center for Genomics, Peking University, Beijing 100871, China;

${ }^{3}$ NHC Key Laboratory of Systems Biology of Pathogens and Christophe Merieux Laboratory, Institute of Pathogen Biology, Chinese Academy Sciences and Peking Union Medical College, Beijing 100730, China;
\end{abstract} \\ of Medical Sciences and Peking Union Medical College; Key Laboratory of Respiratory Disease Pathogenomics, Chinese Academy of Medical \\ ${ }^{4}$ Academy for Advanced Interdisciplinary Studies, Peking University, Beijing 100871, China
}

Received May 28, 2021; accepted August 6, 2021; published online August 20, 2021

\begin{abstract}
The outbreak of coronavirus disease 2019 (COVID-19) caused by SARS-CoV-2 has created a global health crisis. SARS-CoV-2 infects varieties of tissues where the known receptor ACE2 is low or almost absent, suggesting the existence of alternative viral entry pathways. Here, we performed a genome-wide barcoded-CRISPRa screen to identify novel host factors that enable SARSCoV-2 infection. Beyond known host proteins, i.e., ACE2, TMPRSS2, and NRP1, we identified multiple host components, among which LDLRAD3, TMEM30A, and CLEC4G were confirmed as functional receptors for SARS-CoV-2. All these membrane proteins bind directly to spike's N-terminal domain (NTD). Their essential and physiological roles have been confirmed in either neuron or liver cells. In particular, LDLRAD3 and CLEC4G mediate SARS-CoV-2 entry and infection in an ACE2-independent fashion. The identification of the novel receptors and entry mechanisms could advance our understanding of the multiorgan tropism of SARS-CoV-2, and may shed light on the development of COVID-19 countermeasures.
\end{abstract}

CRISPRa screen, SARS-CoV-2, novel receptors

Citation: Zhu, S., Liu, Y., Zhou, Z., Zhang, Z., Xiao, X., Liu, Z., Chen, A., Dong, X., Tian, F., Chen, S., et al. (2022). Genome-wide CRISPR activation screen identifies candidate receptors for SARS-CoV-2 entry. Sci China Life Sci 65, 701-717. https://doi.org/10.1007/s11427-021-1990-5

\section{INTRODUCTION}

The outbreak of coronavirus disease 2019 (COVID-19) has caused a global health crisis. The etiologic agent of COVID-

$\uparrow$ Contributed equally to this work

*Corresponding authors (Junyu Xiao, email: junyuxiao@pku.edu.cn; Jianwei Wang, email: wangjw28@163.com; Wensheng Wei, email: wswei@pku.edu.cn)
19 is acute respiratory syndrome coronavirus 2 (SARS-CoV2), a positive-stranded betacoronavirus (Ren et al., 2020; Zhu et al., 2020). SARS-CoV-2 is the seventh coronavirus known to infect humans, and is the third coronavirus, after severe acute respiratory syndrome (SARS)-CoV and Middle East respiratory syndrome (MERS)-CoV, which has caused outbreaks with significant fatality rates (Andersen et al., 2020). 
SARS-CoV-2 mainly infects the respiratory system, causing symptoms at the onset of disease as fever, cough, fatigue, and myalgia (Huang et al., 2020; Wang et al., 2020). Moreover, COVID-19 is associated with high rates of multiorgan symptoms, such as neurological (Helms et al., 2020), renal (Puelles et al., 2020), gastrointestinal (Cheung et al., 2020), and cardiovascular (Madjid et al., 2020) complications, indicating the broad organotropism of SARS-CoV-2.

Like SARS-CoV, SARS-CoV-2 engages human angiotensin-converting enzyme 2 (ACE2) as the receptor to enter host cells (Zhou et al., 2020). The interaction between SARS-CoV-2 and ACE2 is mediated by the receptor-binding domain (RBD) of the SARS-CoV-2 spike (S) glycoprotein. S protein is cleaved into S1 and S2 domains by cellular proteases such as furin, followed by further cleavage of S2 by proteases such as TMPRSS2 or cathepsins (Hoffmann et al., 2020b; Ou et al., 2020). This "priming" process triggers dramatic conformational changes of the S2 domain to enable the fusion of the viral envelope with cellular membranes, thereby allowing the release of the viral genome into host cells (Hoffmann et al., 2020b; Walls et al., 2020). Despite data showing that ACE2 is a high-affinity receptor for SARS-CoV-2 (Zhou et al., 2020; Shang et al., 2020; Lan et al., 2020), lines of evidence suggested that alternative receptors or pathways may exist. First, the tissue distribution pattern of ACE2 does not fully correlate with SARS-CoV-2 tropism, questioning the ACE2-dependent pathway as the sole entry route. Analyses of the single-cell RNA sequencing data indicated that ACE2 is expressed low throughout the respiratory tract, the primary infection site of SARS-CoV-2 (Aguiar et al., 2020; Lukassen et al., 2020). Moreover, SARS-CoV-2 infects the brain, and viral RNA has been detected in immune cells such as neutrophils, macrophages, T/B cells, and NK cells (Grant et al., 2021; Ren et al., 2021), whereas ACE2 is barely detected in these tissues or cells (Figure S1 in Supporting Information). Second, a recent report showed that an ACE2-null lung adenocarcinoma cell is highly permissive to SARS-CoV-2 (Puray-Chavez et al., 2021), indicating that SARS-CoV-2 could leverage an alternative receptor for its entry. Third, a cell surface protein, AXL, has recently been reported to facilitate SARS-CoV-2 entry independently of ACE2 (Wang et al., 2021). NRP1 was found to function as an ACE2-dependent host factor, which is highly expressed in human pulmonary and olfactory neuronal cells of the epithelium, and could bind to S1 CendR motif of the viral spike protein (Cantuti-Castelvetri et al., 2020; Daly et al., 2020). Altogether, it is plausible to postulate that SARS-CoV-2 may gain its entry to host cells via alternative receptor(s) other than ACE2.

Functional genomics approaches such as CRISPR knockout screens have been conducted to search for critical host factors involved in SARS-CoV-2 infection (Baggen et al., 2021; Daniloski et al., 2021; Wei et al., 2021; Zhu et al.,
2021). However, none of these screens could pinpoint novel receptors beyond ACE2, possibly due to the fact that such loss-of-function screens were performed based on cell types that the expression and function of ACE2 are dominant. Herein, aiming to systematically interrogate host factors for SARS-CoV-2 entry, we performed a genome-wide CRISPR activation screen in HEK293T cells using the SARS-CoV-2 spike-pseudotyped virus (Dieterle et al., 2020). Such gain-offunction screen could potentially identify those proteins that confer host cell susceptibility to SARS-CoV-2.

\section{RESULTS}

\section{A genome-wide barcoded CRISPRa screen identifies novel host components for SARS-CoV-2 infection}

To establish a CRISPRa screening for the identification of viral entry factors, we utilized pseudotyped virus harboring the SARS-CoV-2 spike protein and an EGFP marker that indicates viral infection. EGFP signal was barely detectable two days after infection with different amounts of SARSCoV-2 pseudovirus in HEK293T cells, unlike infection by lentivirus harboring the vesicular stomatitis virus $G$ protein (VSV-G) (Figure S2A in Supporting Information), indicating that SARS-CoV-2 pseudovirus hardly infects HEK293T cells. This was likely due to the lack of sufficient expression of functional receptors in HEK293T cells. Indeed, HEK293 cells stably overexpressing ACE2 were highly susceptible to SARS-CoV-2 pseudovirus, and the EGFP signal was proportionally boosted with the increase of pseudovirus (Figure S2B in Supporting Information). We then tested the effect of gene activation through CRISPRa using the 50-fold concentrated pseudovirus. In HEK293T cells stably expressing CRISPRa system (HEK293T-CRISPRa cells), the upregulation of $A C E 2$ by $\operatorname{sgRNA} 1^{\mathrm{ACE} 2}$ and sgRNA $5^{\mathrm{ACE} 2}$ enabled the infection of SARS-CoV-2 pseudovirus with a significant boost of EGFP expression within the cells (Figure 1A). As such, we developed a CRISPRa screen method to identify host factors enabling SARS-CoV-2 infection.

To reach the optimal performance using the CRISPR activation system (Konermann et al., 2015), we tended to construct a genome-wide CRISPRa library with all sgRNAs barcoded so that we could benefit from a high multiplicity of infection (MOI) in generating the cell library, an approach we previously established (Zhu et al., 2019). Because the loops of sgRNAs are used for such a CRISPRa system we employed (Konermann et al., 2015), we decided to add the barcodes at the external region outside of sgRNA at its $3^{\prime}$ end, designated as eBAR, instead of iBAR we designed before (Zhu et al., 2019). Three external barcodes of $4 \mathrm{nt}$ were assigned to each sgRNA (Figure 1B). The oligos of sgRNA library (Horlbeck et al., 2016) were synthesized and respectively cloned into three lentiviral sgRNA ${ }^{\mathrm{eBAR}}$ back- 
A

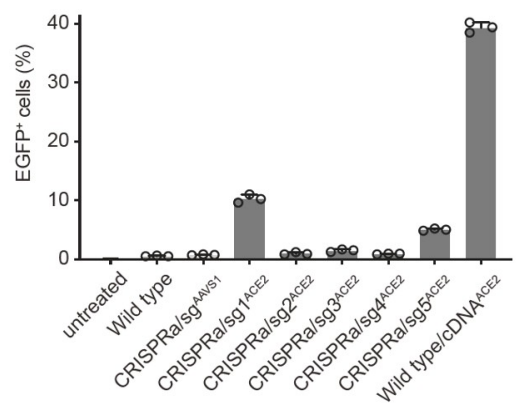

B

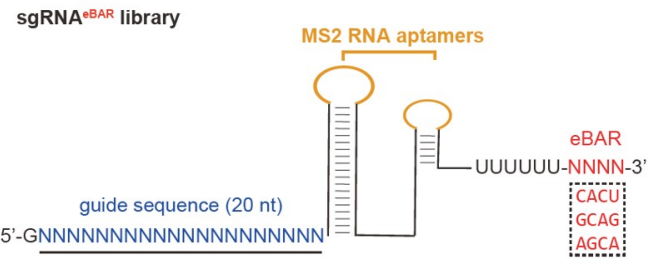

C

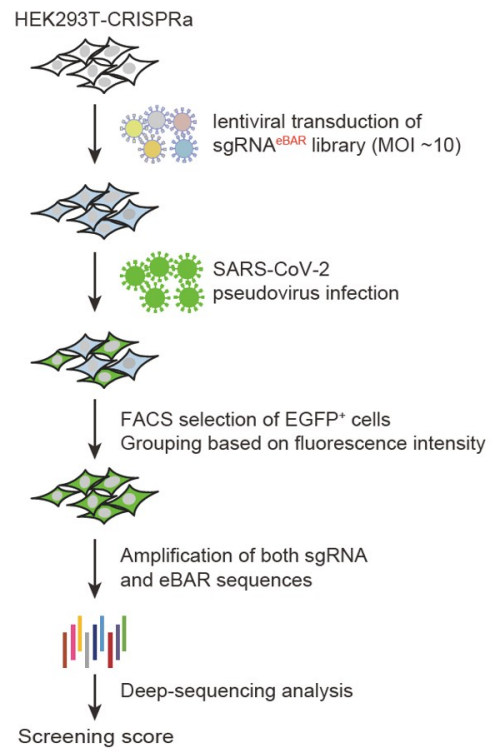

D
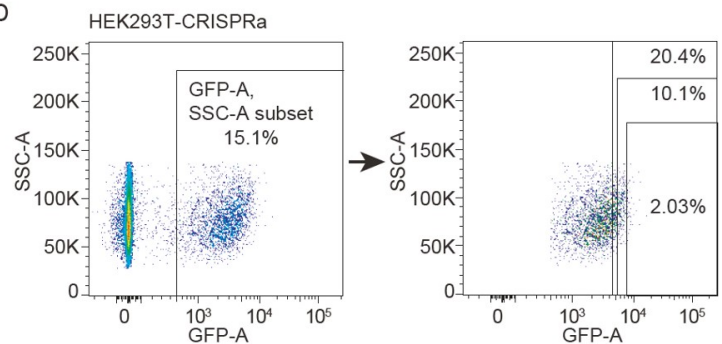

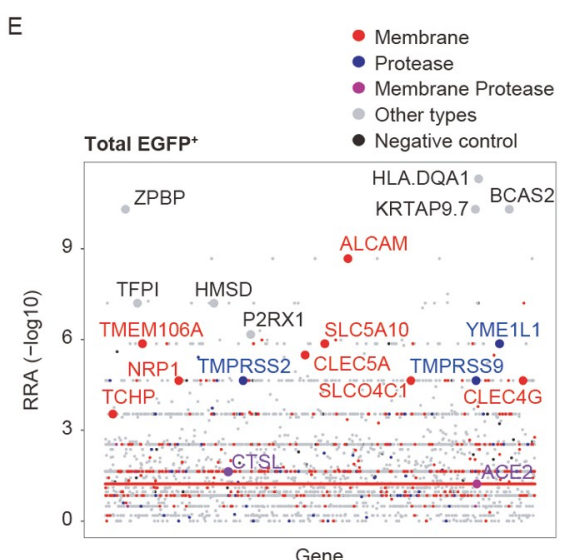

F

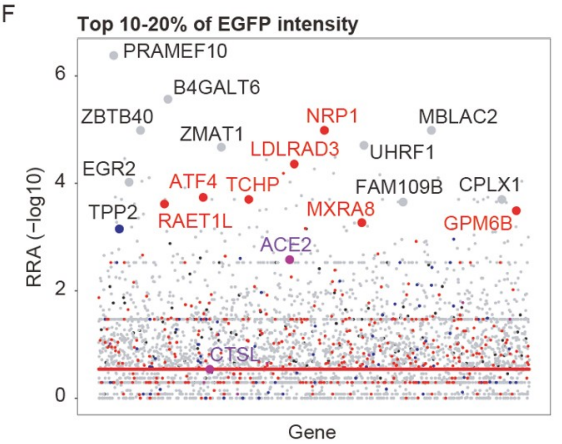

G

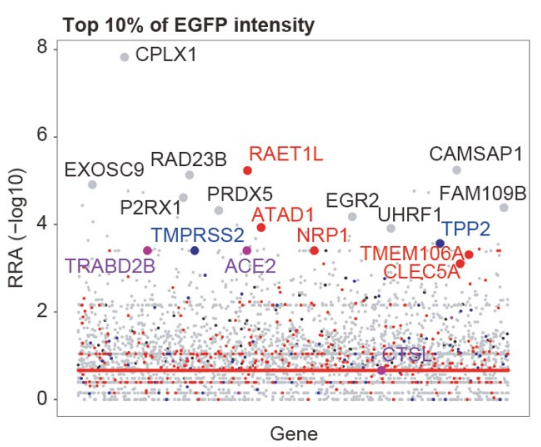

$\mathrm{H}$

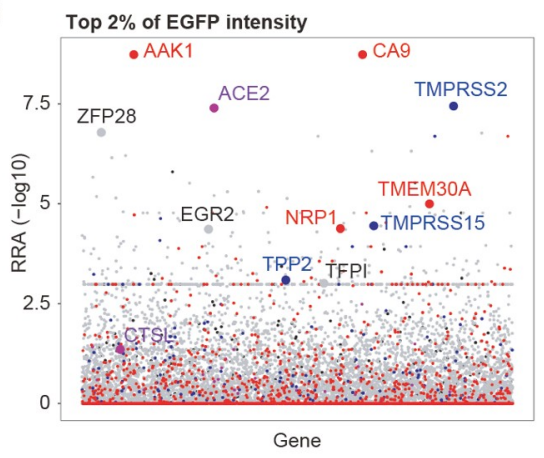

Figure 1 Identification of candidate factors for SARS-CoV-2 entry by a genome-wide CRISPRa gain-of-function screen in HEK293T cells. A, Detection of the pseudovirus infection in HEK293T-CRISPRa cells transfected with different sgRNAs targeting $A C E 2$. The infection rate of SARS-CoV-2 pseudovirus is indicated by the percentage of EGFP-positive cells. Wild type/cDNA ${ }^{\mathrm{ACE} 2}$ represents the wild type HEK293T cells transfected with ACE2 cDNA as a positive control. B, Schematic diagram of an sgRNA with an external barcode (eBAR). Three 4-nt eBARs were respectively embedded outside of the sgRNA scaffold after the poly-U signal. C, Schematic of the CRISPRa screen in HEK293T cells using the SARS-CoV-2 pseudotyped virus. D, FACS selection of EGFP ${ }^{+}$cells grouping based on different fluorescence intensities after SARS-CoV-2 pseudovirus infection. Left indicates the total EGFP intensity of HEK293T-CRISPRa library cells after the second round of pseudovirus infection. Right indicates three additional sorting gates including the top 10\%-20\%, top $10 \%$ and top $2 \%$ of the total $\mathrm{EGFP}^{+}$cells. E-H, Robust rank aggregation (RRA) scores of all genes from the total $\mathrm{EGFP}^{+}(\mathrm{E})$, top $10 \%-20 \%(\mathrm{~F})$, top $10 \%(\mathrm{G})$ and top $2 \%(\mathrm{H})$ of the total $\mathrm{EGFP}^{+}$cells. RRA scores were used to evaluate the enrichment of candidate genes, which were calculated by binomial $P$-values of sgRNAs ${ }^{\mathrm{e} A R}$ targeting each gene. Membrane proteins were labelled as red dots, proteases were labelled as blue dots, the genes that are both membrane protein and proteases were labelled as purple dots. Grey and black dots represent other types of genes and negative controls. 
bones (Table S1 in Supporting Information). The sgRNA ${ }^{\text {eBAR }}$ library was delivered into HEK293T-CRISPRa cells by lentiviral infection at an MOI of $\sim 10$. The pseudovirus (50fold) was added to the library cells, and the infected cells were sorted by FACS (Figure S3A in Supporting Information). Since the EGFP signal was maintained in the sorted cells and could not completely fade out, we were not able to reduce noises through multiple rounds of enrichment. We therefore categorized screening results based on fluorescence intensity, and selected those top candidates from each group to maximize the chance of target identification. After two rounds of pseudovirus infection and sorting, we collected total $\mathrm{EGFP}^{+}$cells as well as top $10 \%-20 \%$, top $10 \%$ and top $2 \%$ of sorted cells grouped by the EGFP intensity (Figure 1C and D; Figure S3B in Supporting Information). We generated screen scores for genes in each $\mathrm{EGFP}^{+}$group considering the performance of all their targeting sgRNAs ${ }^{\mathrm{eBAR}}$ (Figure 1E$\mathrm{H}$; Table S2 in Supporting Information and Methods). In most groups, the known SARS-CoV-2 receptor ACE2 (Lan et al., 2020; Shang et al., 2020; Zhou et al., 2020; Hoffmann et al., 2020b; Walls et al., 2020; Wrapp et al., 2020) and the main host protease TMPRSS2 (Hoffmann et al., 2020b) were significantly enriched. We also identified other reported host factors for SARS-CoV-2 entry, such as NRP1 (CantutiCastelvetri et al., 2020; Daly et al., 2020). The viral protease CTSL identified in multiple groups is a known factor that can substitute TMPRSS2 to prime the SARS-CoV spike for membrane fusion (Simmons et al., 2013), and was also reported to be critical in the endosomal fusion pathway for SARS-CoV-2 entry (Zhu et al., 2021). The EGFP intensity was supposed to represent the strength of the target host factor in promoting virus entry. Thus we assumed that receptors were more likely to be identified from groups with higher EGFP intensity. For example, ACE2 was ranked higher in the top $2 \%$ than in other groups (Figure 1E-H).

To further characterize these identified host factors, we performed Gene Ontology (GO) enrichment analysis (Zhou et al., 2019). A number of genes were enriched in multiple important cellular processes, such as regulation of plasma membrane-bound cell projection organization, vesiclemediated transport, receptor-mediated endocytosis, and viral life cycle (Figure 2A; Figure S4 and Table S3 in Supporting Information). Many of these genes were top-ranked in most groups of the sorted $\mathrm{EGFP}^{+}$cells (Figure 2B). Assuming that the intensity of EGFP represented the strength of candidate factors in facilitating virus entry, we were particularly interested in membrane proteins identified from the top $2 \%$ and $10 \%$ groups. For other types of candidates, we pooled topranked candidates in all four groups for validation. For each gene, we found that most of its corresponding sgRNAs ${ }^{\text {eBAR }}$ were significantly enriched, indicating the reliability of our selection on the top hits. Besides, most of the functional sgRNAs performed consistently with their eBARs (Figure
S5 in Supporting Information). The gene expression analysis revealed that several genes are widely expressed in multiple tissues such as TMEM3OA and CTSL, and some genes' expressions are more tissue-specific, such as brain-specific genes $C P L X 1, L D L R A D 3, G P M 6 B$ and $E P H B 1$, liver-specific genes CLEC4G and MASP1, lung-specific genes CLEC5A and HLA-DQA1, and immune-specific genes ICAM2 and STAMBPL1 (Figure 2C). These findings hold the potential to interpret the organotropism of SARS-CoV-2 especially where the known receptors and other entry factors were lowly expressed.

\section{Validation of identified genes through SARS-CoV-2 pseudotyped and authentic virus}

To validate the candidate genes identified from our screen, we focused particularly on membrane proteins, proteases, and some other top-ranked hits. For a total of 51 candidates, we transduced HEK293T cells with their corresponding cDNAs, followed by infection with SARS-CoV-2 pseudotyped virus containing a luciferase reporter (Dieterle et al., 2020). As the known receptor or co-receptor for SARS-CoV2 , the ectopic expression of ACE2 or NRP1 greatly promoted the pseudotyped virus infection (Figure 3A). A number of novel host factors have been confirmed to facilitate SARSCoV-2 pseudovirus entry, including some membrane proteins, LDLRAD3, TMEM30A, CLEC4G, CPLX1, and CA9 (Figure 3A). LDLRAD3 is a member of the LDL scavengerreceptor family that is highly expressed in neurons and has been reported to regulate amyloid precursor protein in neurons (Ranganathan et al., 2011). TMEM30A is a transmembrane protein involved in membrane trafficking and signaling pathways as a heterocomplex with ATP8A1 by regulating the translocation of phospholipids (Hiraizumi et al., 2019). CLEC4G is a member of the C-type lectin family that has been reported to enhance the infection of SARS$\mathrm{CoV}$ by interacting with its spike protein (Gramberg et al., 2005). CPLX1 is a member of the complexin/synaphin family involved in synaptic vesicle exocytosis and transmitter release (Karaca et al., 2015). CA9, a transmembrane protein and a tumour marker (Al-Sharaky et al., 2020), has also been reported to be involved in HBV infection (Fan et al., 2020). Interestingly, two proteases, STAMBPL1 and TMPRSS15, were also identified with their confirmed roles to promote SARS-CoV-2 pseudovirus infection (Figure 3A). Proteases such as TMPRSS2 are known to play critical roles in ACE2dependent virus entry (Hoffmann et al., 2020b). Therefore, we reasoned that proteases with similar functions could promote virus entry upon overexpression. We went on to validate these candidate genes using the authentic SARSCoV-2 virus. In HEK293T cells, besides ACE2 and CTSL, the ectopic expression of any of the following genes could effectively enable SARS-CoV-2 infection, CLEC4G, 


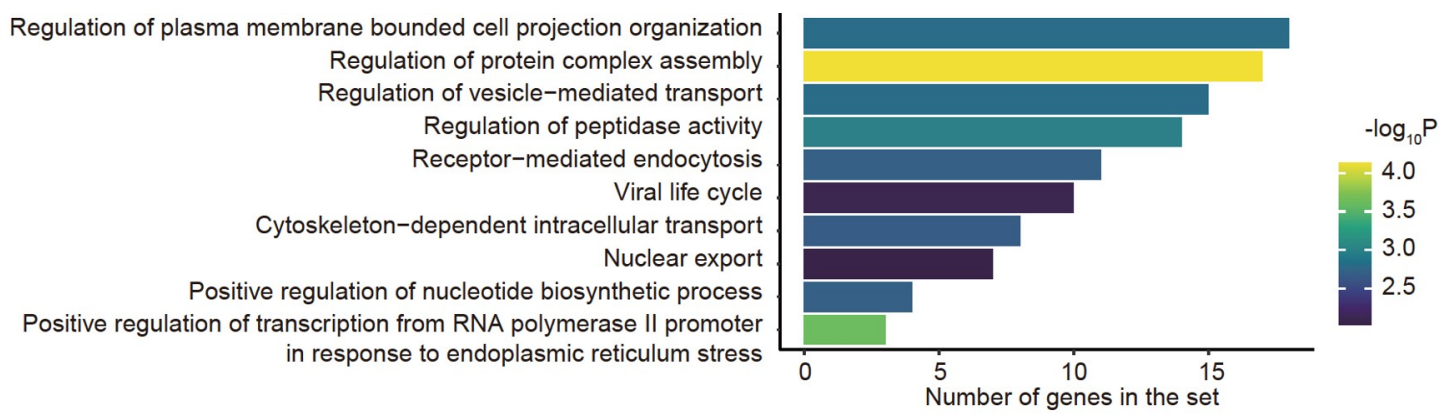

B

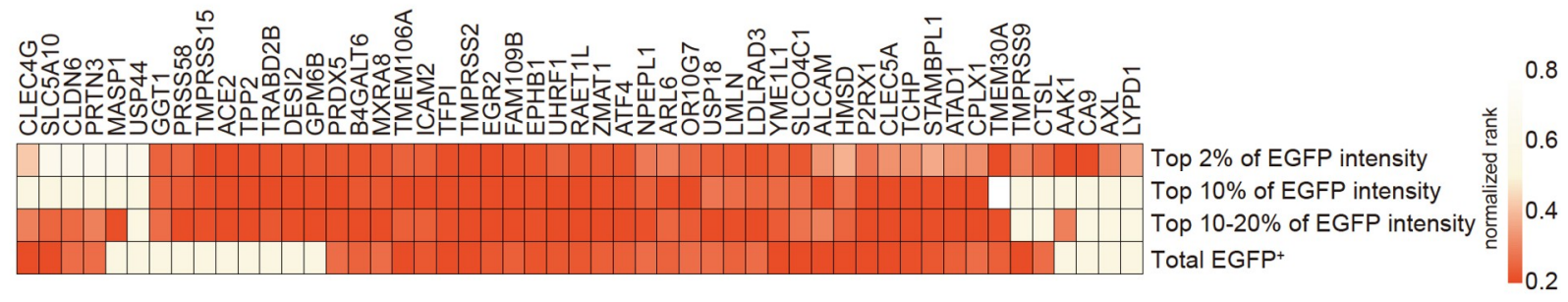

C

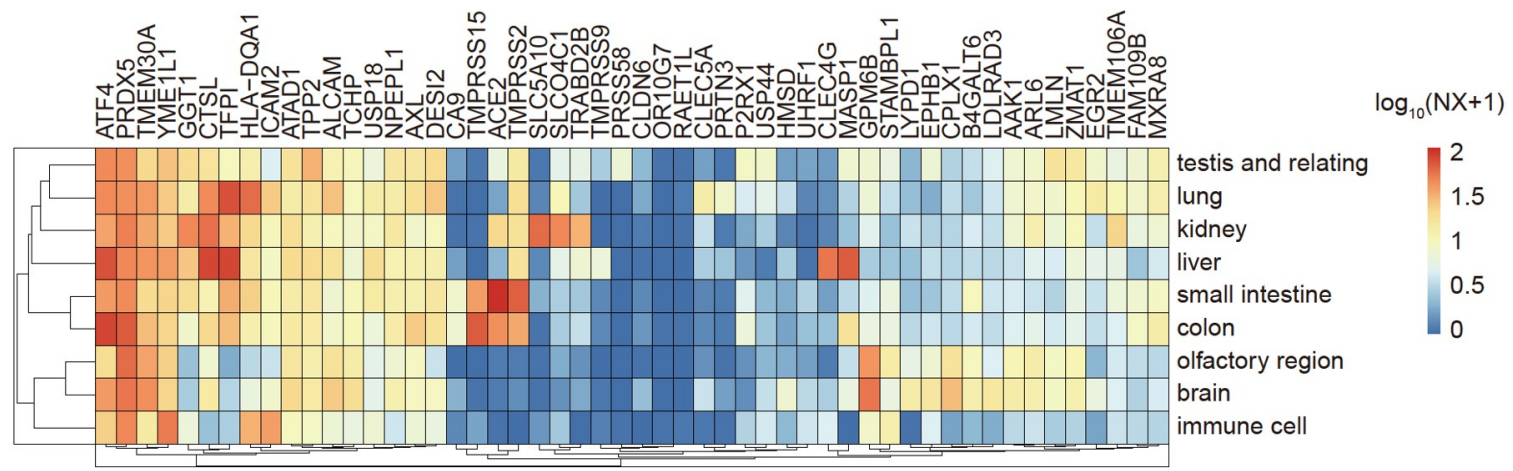

Figure 2 Host factors identified from CRISPRa library screening. A, Gene Ontology (GO) enrichment analysis was conducted using all the significant candidates (RRA score $<0.001$ ) identified in the four groups. Hypergeometric test was used to calculate all the $P$-values. The top-enriched GO terms were selected for visualization. The $x$ axis represents the number of genes identified in the specific GO terms. A complete list of genes in each GO term is in Table S3 in Supporting Information. B, The performance of all the significant hits in four screening groups (top $2 \%$, top $10 \%$, top $10 \%-20 \%$ of EGFP intensity and total EGFP ${ }^{+}$). The gene with a smaller value of normalized rank (in redder colour) represented a higher enrichment in the relevant groups. C, Expression patterns of identified candidates within human tissues. The data used for analysis were retrieved from the Human Protein Atlas normalized expression.

CPLX1, LDLRAD3, TMEM3OA, and STAMBPL1 (Figure 3B).

To examine if ACE2 is required for any of these candidate components to promote viral infection, we generated HEK293T $A C E 2^{-/-}$cells (Figure S6 in Supporting Information). We found that the function of CA9, CLEC4G, and LDLRAD3 in facilitating luciferase reporter pseudovirus infection is independent of ACE2 (Figure 3C). In the test of authentic virus infection, overexpression of either CLEC4G or LDLRAD3 is sufficient to enable SARS-CoV-2 infection in HEK293T $A C E 2^{-/}$cells (Figure 3D).

\section{LDLRAD3, CLEC4G and TMEM30A bind NTD of SARS-CoV-2 spike protein directly}

Next, we focused on characterizing these membrane proteins and evaluating whether any of them serves as a functional receptor for SARS-CoV-2. We first examined whether there are interactions between these receptor candidates and SARS-CoV-2 spike (S) protein. Co-immunoprecipitation (Co-IP) assay showed that SARS-CoV-2 S co-precipitated with multiple candidate proteins including TMEM30A, ICAM2, CA9, LDLRAD3, CLEC4G, and the known host factors, ACE2, NRP1, TMPRSS2 and CTSL, but not with STAMBPL1 and CPLX1 (Figure 4A). We then purified these proteins (Figure S7A in Supporting Information) to examine the direct interactions by the pull-down assay. Like ACE2, LDLRAD3 and CLEC4G efficiently pulled down SARSCoV-2 S (Figure 4B). Reciprocally, SARS-CoV-2 S pulled down LDLRAD3, CLEC4G and ACE2, but not CA9 (Figure S7B in Supporting Information). Moreover, we determined domains on SARS-CoV-2 S that mediate the interactions. In consistence with previous reports (Zhou et al., 2020), ACE2 interacted with RBD but not NTD (Figure 4D and C). However, NTD but not RBD were found to directly interact with LDLRAD3, CLEC4G, and TMEM30A (Figure 4C and 

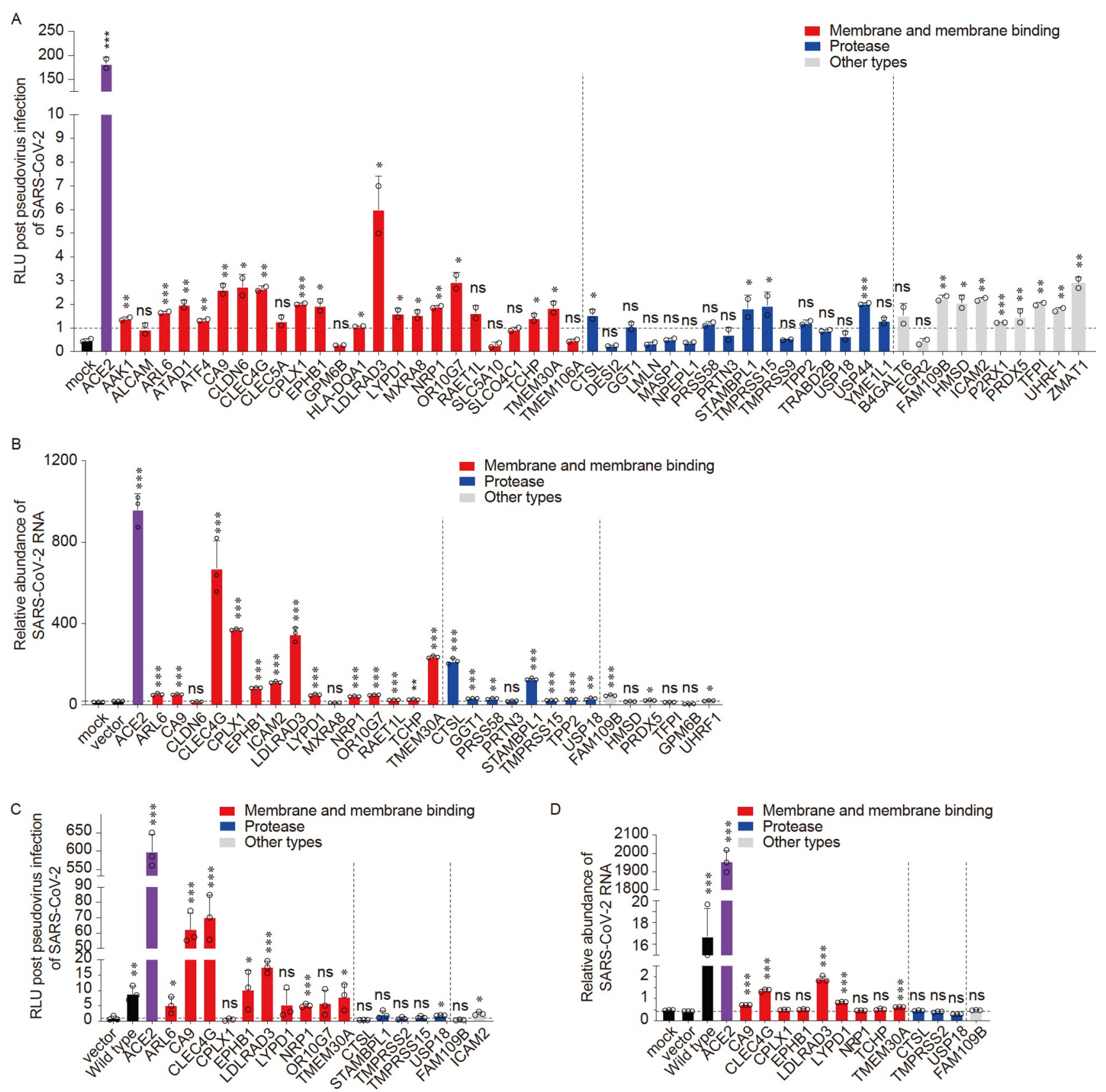

Figure 3 Validation of candidate genes identified from CRISPRa library screening. A and B, Effects of identified genes on the infection of SARS-CoV-2. A, 51 individual cDNAs and an empty vector were transfected into HEK293T cells. Then the cells were treated with luciferase-labelled SARS-CoV-2 pseudotyped virus. The entry of SARS-CoV-2 pseudotyped virus was quantified through measuring luciferase activity $48 \mathrm{~h}$ later. The luciferase activities were normalized by the empty vector. Data are presented as the mean \pm SD $(n=2)$. B, The cDNAs of candidate genes were introduced into HEK293T cells lentivirally labelled with an mCherry marker. The mCherry-positive cells were enriched through FACS followed by infection with authentic SARS-CoV-2 virus at an MOI of 0.5 . SARS-CoV-2 RNAs were quantified by real-time qPCR and normalized by $G A P D H$. Data were presented as the mean \pm SD $(n=3)$. C and D, Effects of identified genes on the infection of SARS-CoV-2 in $A C E 2^{-/-}$cells. C, The cDNAs were transfected into HEK293T $A C E 2^{-/-}$cells. Then the cells were treated with 10-fold concentrated SARS-CoV-2 pseudotyped virus. The entry of pseudotyped virus was quantified through measuring luciferase activity and was normalized by the empty vector. D, The cDNAs of candidate genes were introduced into HEK293T $A C E 2^{-/-}$cells lentivirally. Cells were enriched through FACS followed by infection with authentic SARS-CoV-2 virus at an MOI of 0.5. SARS-CoV-2 RNAs were quantified by real-time qPCR and normalized by $G A P D H$. Data were presented as the mean \pm SD $(n=3)$. $P$ values were calculated using Student's $t$ test, $* P<0.05 ; * * P<0.01 ; * * * P<0.001$; ns, not significant.

D). Surface plasmon resonance (SPR) analysis further determined the interaction kinetics, showing an equilibrium dissociation constant of $94 \mathrm{nmol} \mathrm{L}^{-1}$ for ACE2, $293 \mathrm{nmol} \mathrm{L}^{-1}$ for LDLRAD3, and $282 \mathrm{nmol} \mathrm{L}^{-1}$ for CLEC4G (Figure 4E). In light of these direct binding results, we predicted that the extracellular addition of these purified proteins could 
A

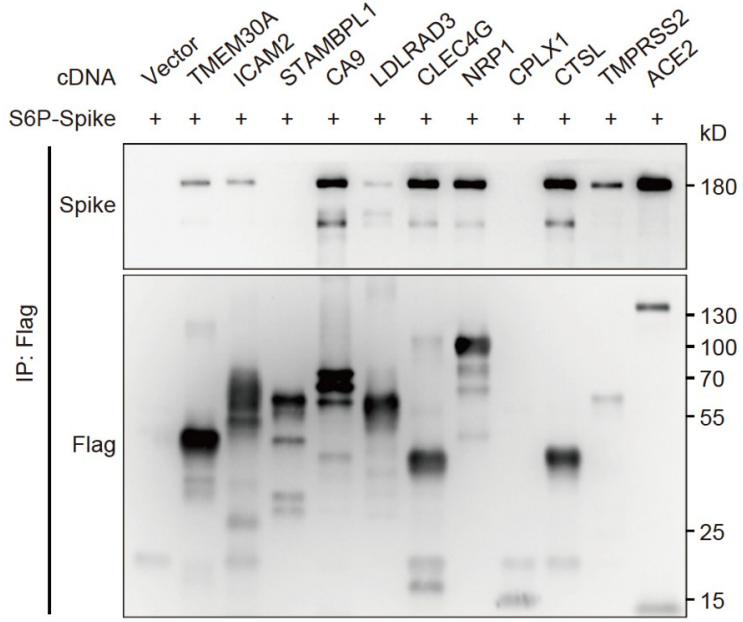

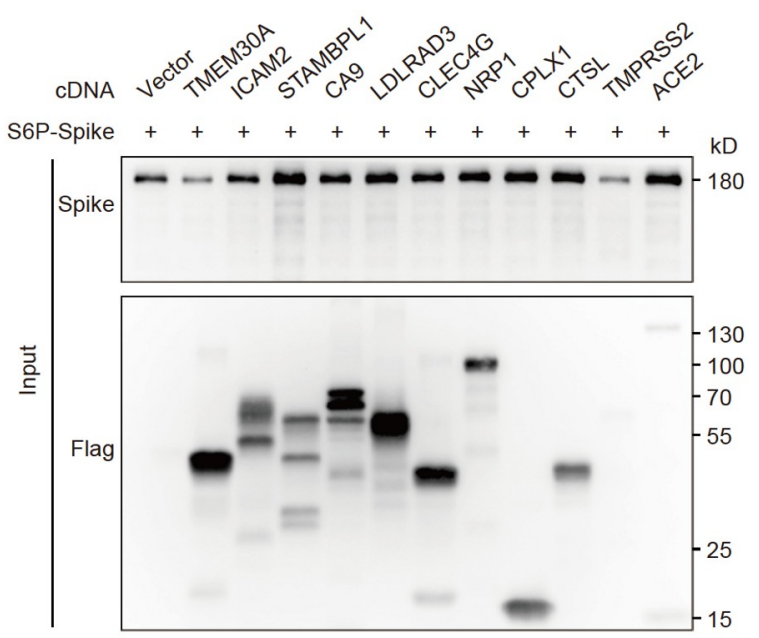

B
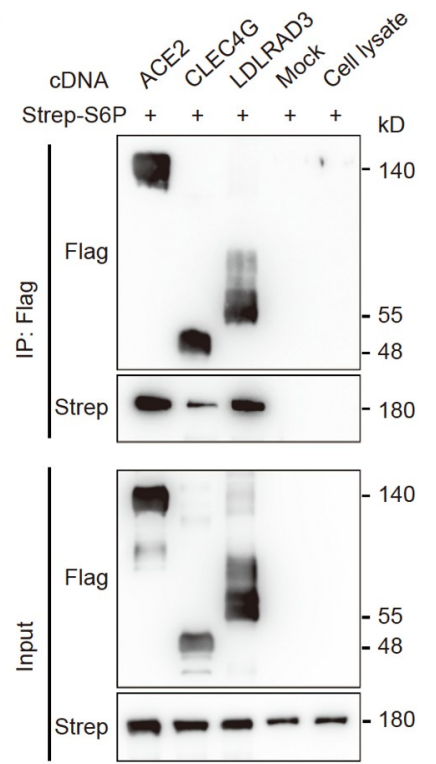

E

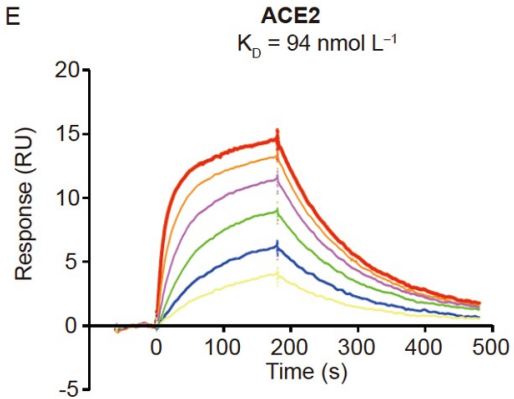

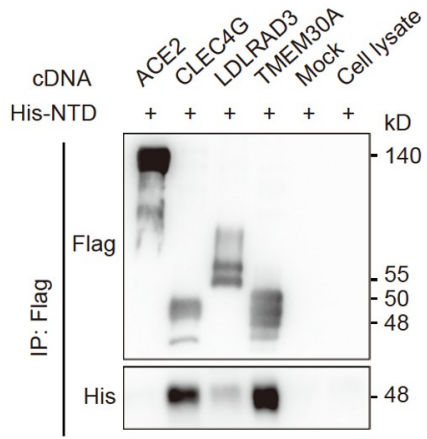

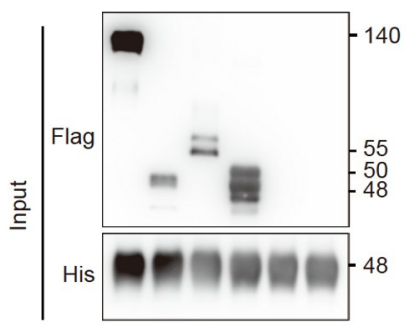

LDLRAD3

$K_{D}=293 \mathrm{nmol} \mathrm{L}^{-1}$

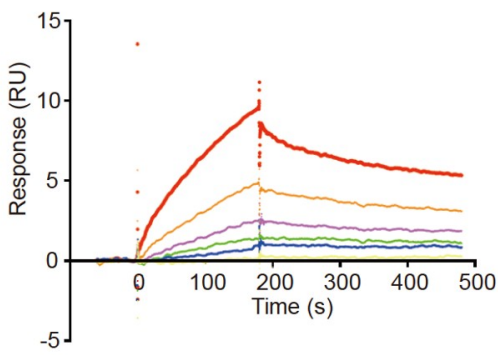

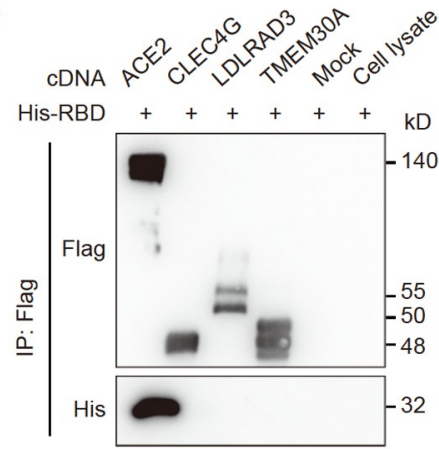

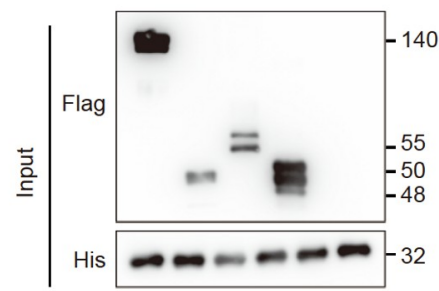

CLEC4G

$\mathrm{K}_{\mathrm{D}}=282 \mathrm{nmol} \mathrm{L}^{-1}$

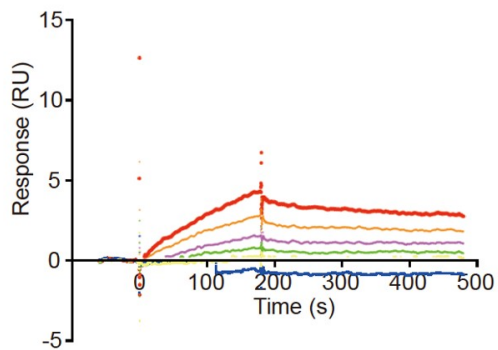

Figure 4 Direct binding of LDLRAD3, CLEC4G and TMEM30A to SARS-CoV-2 S. A, Co-IP of SARS-CoV-2 S6P spike with FLAG-tagged proteins in HEK293T cells transfected with Flag-cDNA constructs and SARS-CoV-2 S. Immunoblot analysis was conducted using anti-Flag and anti-spike antibodies. B, In vitro pull-down assay of purified ACE2, CLEC4G and LDLRAD3 to SARS-CoV-2 S. Strep-tagged SARS-CoV-2 S and FLAG-tagged full-length candidate receptors were expressed in HEK293T cells and affinity-purified. Immunoblot analysis was conducted using anti-Flag and anti-Strep antibodies. C and D, In vitro pull-down assay of purified ACE2, CLEC4G, LDLRAD3 and TMEM30A to the NTD (C) or RBD (D) of SARS-CoV-2 S. Immunoblot analysis was conducted using anti-Flag and anti-His antibodies. E, Surface plasmon resonance (SPR) measurements for the dynamic binding between candidate receptors and SARS-CoV-2 S. Purified extracellular domain of ACE2, full-length of LDLRAD3 and CLEC4G $(0.03125,0.0625,0.125,0.25,0.5$ and $1 \mu \mathrm{mol} \mathrm{L}{ }^{-1}$ ) were tested.

prevent virus entry by competing cellular receptors for binding to $\mathrm{S}$. To test this idea, we incubated serially diluted soluble proteins with authentic SARS-CoV-2 virus before infection. The addition of soluble ACE2 (Figure 5A and B) 

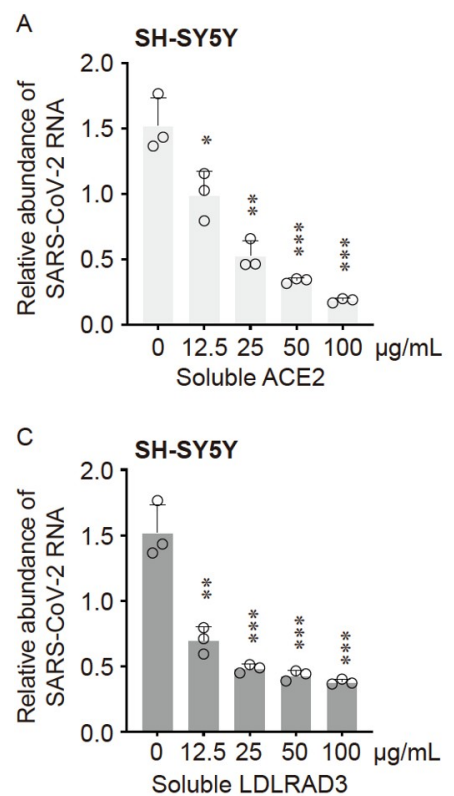
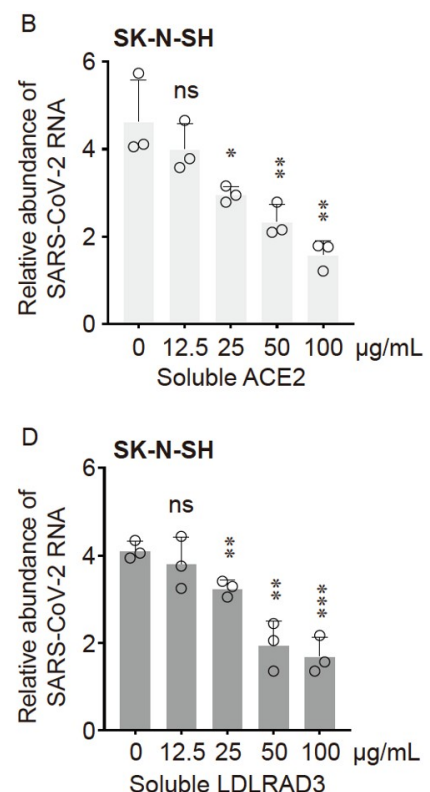
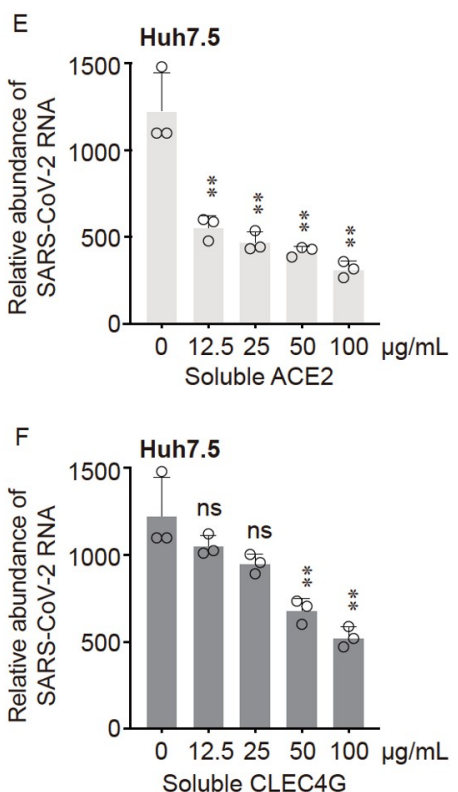

Figure 5 Inhibition of soluble proteins on SARS-CoV-2 infection. A and B, Effects of purified ACE2 on SARS-CoV-2 infection in SH-SY5Y (A) and SKN-SH (B) cells. C and D, Effects of purified LDLRAD3 on SARS-CoV-2 infection in SH-SY5Y (C) and SK-N-SH (D) cells. E and F, Effects of purified ACE2 (E) and CLEC4G (F) on SARS-CoV-2 infection in Huh7.5 cells. The soluble proteins $\left(0,12.5,25,50\right.$ and $\left.100 \mu \mathrm{g} \mathrm{mL}^{-1}\right)$ were incubated with authentic SARS-CoV-2 virus for $1 \mathrm{~h}$. Infection was performed at an MOI of 0.5. SARS-CoV-2 RNAs were quantified by real-time qPCR and normalized by GAPDH. Data were presented as the mean $\pm \mathrm{SD}(n=3)$. $P$ values were calculated using Student's $t$ test, ${ }^{*} P<0.05$; $* * P<0.01 ; * * * P<0.001$; ns, not significant.

and LDLRAD3 (Figure 5C and D) were capable of protecting both SH-SY5Y (Figure 5A and C) and SK-N-SH (Figure 5B and D), two neuroblastoma cell lines, from SARS-CoV-2 infection, in a dose-dependent manner. Similarly, the addition of soluble ACE2 (Figure 5E) and CLEC4G (Figure 5F) effectively suppressed SARS-CoV-2 infection in a liver cancer cell line Huh7.5, also in a dose-dependent manner.

\section{LDLRAD3, CLEC4G and TMEM30A trigger mem- brane-to-membrane fusion through interaction with SARS-CoV-2 spike protein}

SARS-CoV-2's entry is initiated by the interaction between the spike and its host receptor(s), followed by furin-mediated cleavage at the S1/S2 site and the priming via TMPRSS2 or other surface/endosomal proteases (Hoffmann et al., 2020a; Matsuyama et al., 2020). The surface subunit $\mathrm{S} 1$ of spike is responsible for binding to the host receptor, and the transmembrane subunit S2 mediates the viral and cellular membrane fusion (Hoffmann et al., 2020b). Previous studies have shown that SARS-CoV-2 S present in the plasma membrane possesses high fusogenic activity and could trigger receptordependent fusion with neighboring cells, leading to the formation of multinucleated giant cells (syncytia) (Buchrieser et al., 2021; Hoffmann et al., 2020a). To examine whether the interaction between the SARS-CoV-2 spike protein and our newly identified receptors could elicit membrane fusion, we performed a co-culture assay to determine the syncytium formation. The wild-type HEK293T cells transfected with plasmids expressing $\mathrm{S}$ and EGFP were mixed with HEK293T cells stably overexpressing individual candidate receptors labelled with an mCherry marker (see Methods). At $40 \mathrm{~h}$ post cell co-culture, cells expressing any of the following, ACE2, CLEC4G, LDLRAD3 and TMEM $30 A$, substantially fused with cells expressing $\mathrm{S}$, manifested by the colocalization of the EGFP and mCherry fluorescent signals in the merged images, which could be visualized even in the bright field (Figure 6). In comparison, the control cells infected with only the empty vector showed no syncytium formation, nor merged fluorescent signals (Figure 6). In ACE2-null HEK293T cells, overexpressing CLEC4G, LDLRAD3, and TMEM3OA also showed abundant syncytia, albeit to a lesser extent as compared with $A C E 2$ (Figure $\mathrm{S} 8$ in Supporting Information). These observations suggested that any of CLEC4G, LDLRAD3 and TMEM30A functionally interacts with spike protein of SARS-CoV-2 to trigger membrane-to-membrane fusion, a critical step for receptormediated viral entry, just as ACE2.

\section{Loss-of-function investigation of identified receptors in neuron and liver cell lines}

To evaluate their physiological roles, we first conducted expression analysis using the Human Protein Atlas (HPA) (Uhlén et al., 2015). LDLRAD3 is preferentially expressed in brain tissue, such as cerebellum, spinal cord and salivary gland (Figure S9A in Supporting Information). The expres- 

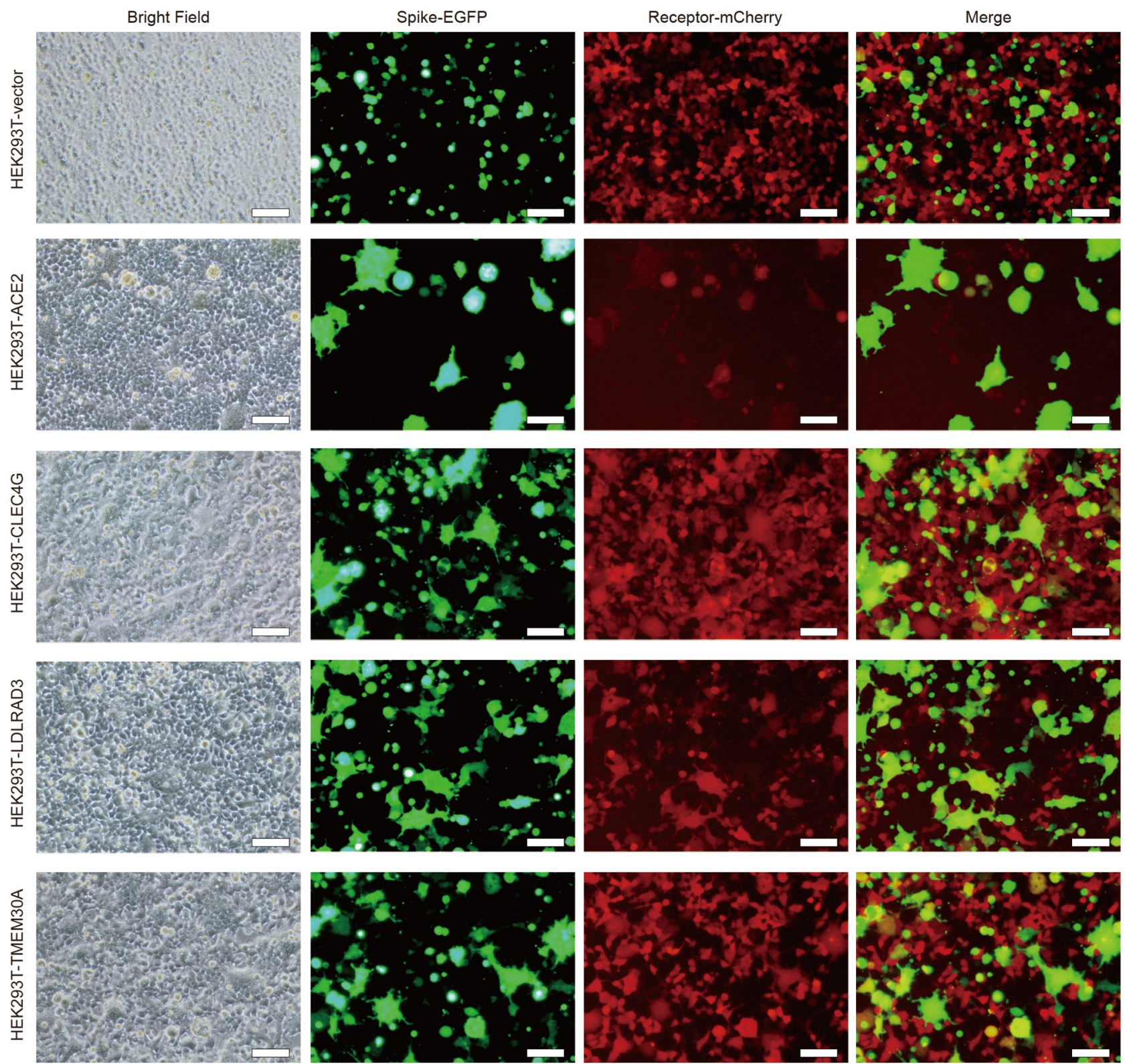

Figure 6 Examination of the interaction between SARS-CoV-2 S protein and candidate receptors by syncytium formation assay. Spike-EGFP represents the HEK293T cells transfected with SARS-CoV-2 S protein and an EGFP marker. Receptor-mCherry represents the HEK293T cells stably overexpressed with the known and candidate receptors labelled with an mCherry marker, labeled as HEK293T-ACE2, HEK293T-CLEC4G, HEK293T-LDLRAD3 and HEK293TTMEM30A. HEK293T cells infected with the cDNA-expressing vector, labelled as HEK293T-vector, was served as the control. Merge indicates the colocalization of the two categories of cells through merging the EGFP and mCherry fluorescence channels by ImageJ. The images were taken $40 \mathrm{~h}$ after coculturing the two categories of cells. Scale bar, $100 \mu \mathrm{m}$.

sion of CLEC4G could only be detected in liver and lymph node (Figure S9B in Supporting Information). While TMEM30A is more ubiquitously expressed in tissues including those with a high incidence of infection, such as lung, colon and airway (Figure S9C in Supporting Information). We then tested whether these candidate receptors are required for SARS-CoV-2 in specific cells. TMEM3OA and LDLRAD3 showed much higher expression compared to $A C E 2$ in $\mathrm{SH}-$ SY5Y cells, which is consistent with the analysis from HPA (Figure 7A). The siRNAs targeting ACE2, TMEM30A, and $L D L R A D 3$ were introduced into indicated cells followed by authentic SARS-CoV-2 infection (Table S4 in Supporting Information). Efficient knockdown was confirmed by qPCR analysis (Figure 7B-D). The disruption of ACE2, LDLRAD3 and TMEM30A expression all led to significant cellular resistance to SARS-CoV-2 infection (Figure 7E). Of note, the extent of siRNA knockdown correlated well with the inhibitory effects to the viral infection (Figure 7E). Similar in SH-SY5Y, LDLRAD3 and TMEM30A were highly expressed in another neuron cell line, SK-N-SH (Figure 7F). Moreover, the expression of $A C E 2$ in SK-N-SH was too low to be detected through qPCR. The siRNA knockdown of LDLRAD3 
Zhu, S., et al. Sci China Life Sci April (2022) Vol.65 No.4
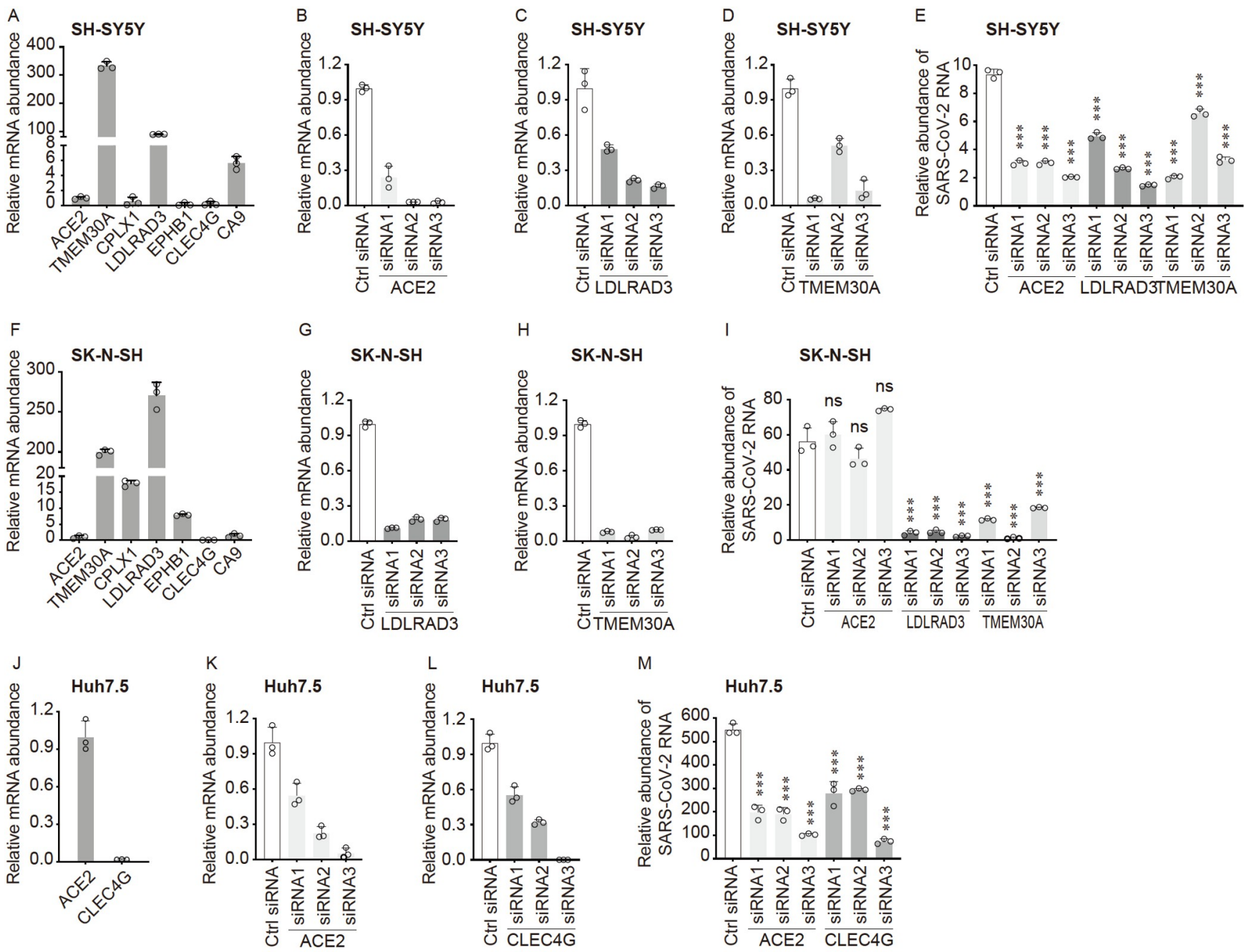

Figure 7 Loss-of-function effects of identified receptors on SARS-CoV-2 infection. A, Expression of identified host factors relative to ACE2 in SH-SY5Y cells. B-D, Suppression of $A C E 2$ (B), LDLRAD3 (C) and TMEM30A (D) by siRNAs in SH-SY5Y cells. E, Effects of suppression of candidate receptors by siRNAs on SARS-CoV-2 infection in SH-SY5Y cells. Infection was performed at an MOI of 0.5. F, Expression of identified host genes relative to $A C E 2$ in SK-N-SH cells. G and H, Suppression of $L D L R A D 3(\mathrm{G})$ and TMEM30A $(\mathrm{H})$ by siRNAs in in SK-N-SH cells. I, Effects of suppression of candidate genes by siRNAs on SARS-CoV-2 infection in SK-N-SH cells. Infection was performed at an MOI of 0.5. J, Expression of identified host genes relative to $A C E 2$ in Huh7.5 cells. K and L, Suppression of $A C E 2(\mathrm{~K})$ and $C L E C 4 G(\mathrm{~L})$ by siRNAs in in Huh7.5 cells. M, Effects of suppression of candidate genes by siRNAs on SARS-CoV-2 infection in Huh7.5 cells. Infection was performed at an MOI of 0.5. For all these experiments, a total of 20 pmol for each siRNA was transfected into cells. The relative mRNA abundance was quantified $48 \mathrm{~h}$ post transfection. Ctrl RNA: Random non-targeting siRNA. RNA abundance of host factors and SARS-CoV-2 were quantified by real-time qPCR and normalized by $G A P D H$. Data were presented as the mean \pm SD $(n=3$ ). $P$ values were calculated using Student's $t$ test, ${ }^{*} P<0.05$; $* * P<0.01$; $* * * P<0.001$; ns, not significant. All siRNAs used for gene suppression are listed in Table S4 in Supporting Information, and primers used for real-time qPCR are listed in Table S5 in Supporting Information.

and TMEM3OA (Figure $7 \mathrm{G}$ and $\mathrm{H}$ ) significantly blocked SARS-CoV-2 infection, whereas ACE2-targeting siRNAs exerted no effects, likely due to the lack of endogenous $A C E 2$ expression (Figure 7I). As CLEC4G is preferentially expressed in the liver, we tested its function in Huh7.5 cells. The qPCR results indicated a lower expression level of $C L E C 4 G$ than $A C E 2$ in Huh7.5 (Figure 7J). Nevertheless, knockdown of either $A C E 2$ or $C L E C 4 G$ (Figure $7 \mathrm{~K}$ and $\mathrm{L}$ ) significantly inhibited SARS-CoV-2 infection in Huh7.5 cells (Figure $7 \mathrm{M}$ ). These results clearly demonstrated the essential roles of LDLRAD3, TMEM30A and CLEC4G in SARS-CoV-2 infection, especially in cell types where ACE2 is lowly expressed.

\section{Physiological roles of novel identified receptors for SARS-CoV-2 infection}

To gain further insights into the physiological relevance of identified receptors for SARS-CoV-2 infection, we analysed the expression of ACE2, LDLRAD3, CLEC4G, and TME$M 30 A$, together with abundances of viral genes in a singlecell RNA-sequencing profile of the airways of 32 COVID-19 patients $(78,264$ cells $)$ and 16 SARS-CoV-2-negative control donors (9,913 cells) (Trump et al., 2021). The expressions of $A C E 2$ (Figure 8A), LDLRAD3 (Figure 8B), CLEC4G (Figure $8 \mathrm{C}$ ), and TMEM30A (Figure 8D) are correlated with SARSCoV-2 RNA levels. Among SARS-CoV-2-positive cells, 
A

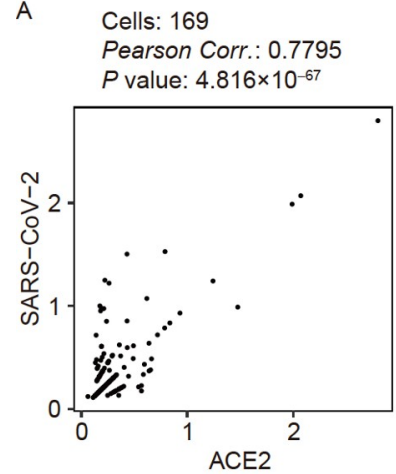

E

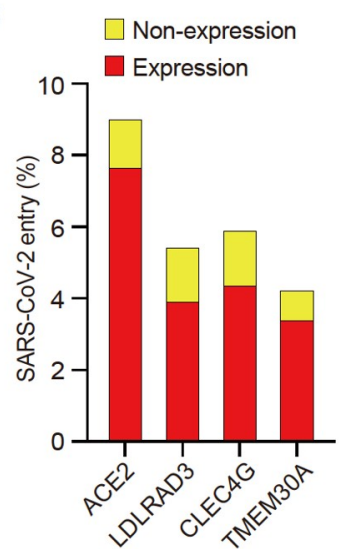

B

\section{Cells: 40}

Pearson Corr.: 0.2283

$P$ value: $9.084 \times 10^{-8}$

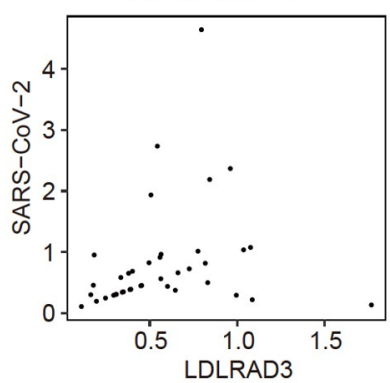

C

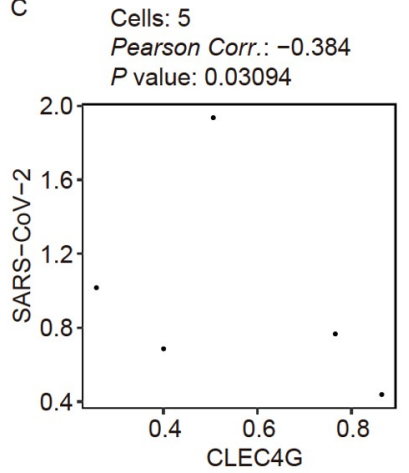

D

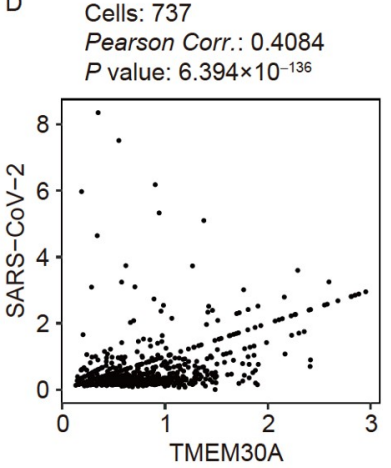

F

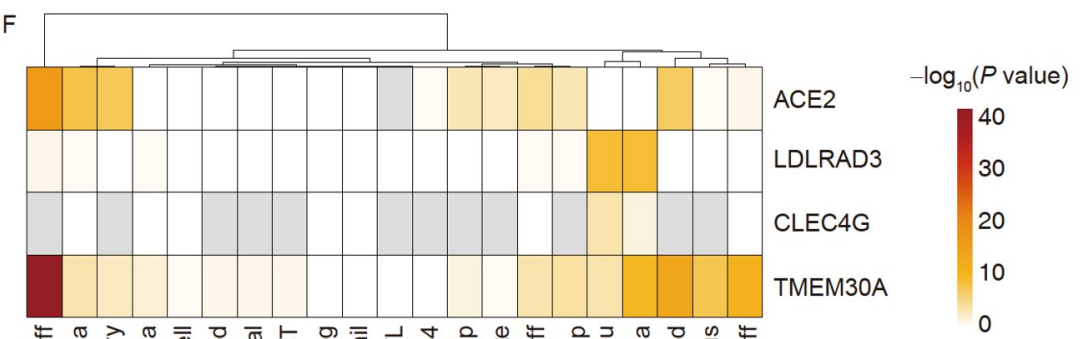

Figure 8 Expression of candidate receptors in nasopharyngeal samples from COVID-19 patients correlates with SARS-CoV-2 infection. A-D, Evaluation of the co-expression between SARS-CoV-2 and ACE2 (A), LDLRAD3 (B), CLEC4G (C) or TMEM30A (D) from COVID-19 patients at the single-cell level. $\mathrm{E}$, The percentage of SARS-CoV-2 entry in single cells with/without expression of each candidate gene. F, Evaluation of the co-expression between SARSCoV-2 and each candidate gene in different cell types from COVID-19 patients at the single-cell level. The $P$ value shown in each figure (A-D, F) was calculated by the Fisher test to evaluate the significance of co-expression between SARS-CoV-2 and each candidate receptor.

there are more TMEM30A-expressing cells (737 of 1,205 cells) than $A C E 2$-expressing cells (169 of 1,205 cells) (Figure $8 \mathrm{~A}$ and $\mathrm{D}$ ). We then grouped cells into candidate geneexpression cells and non-expression cells, and calculated the percentages of SARS-CoV-2 infection in these two groups. For all these four genes, the SARS-CoV-2 infection percentages are much higher in expression cells than non-expression cells (Figure 8E). The lowest SARS-CoV-2 infection percentage was observed in cells that do not express TMEM30A compared to cells that do not express $A C E 2$, LDLRAD3, and CLEC4G, showing its potential importance in mediating SARS-CoV-2 entry (Figure $8 \mathrm{E}$ ). The expression of TMEM $30 A$ and $A C E 2$ significantly correlate with SARS-CoV-2 infection in epithelial sub-clusters such as Ciliated-diff (differentiating), Ciliated, and Secretory cells, while $L D L R A D 3$ and $C L E C 4 G$ expression correlate with viral infection predominantly in immune cells like Neu (neutrophil) and rMa (resident macrophages) cell types (Figure 8F; Figure S10 in Supporting Information). These data demonstrate the relevance of identified receptors with SARS-CoV-2 infectivity in airway cells of COVID-19 patients.

\section{DISCUSSION}

Here we conducted a study of applying a gain-of-function screen for SARS-CoV-2 entry, which uncovered three new viral receptors besides ACE2. Of the candidate receptors identified in this study, LDLRAD3 is highly expressed in neurons, and its overexpression robustly enhanced SARSCoV-2 infection in both wild-type and HEK293T $A C E 2^{-/-}$ cells. LDLRAD3 has recently received attention as a critical receptor for the Venezuelan equine encephalitis virus (VEEV) (Ma et al., 2020). Similar to VEEV, SARS-CoV-2 was also reported to infect the brain (Puelles et al., 2020). Our data revealed that knockdown of LDLRAD3 or supplement of its soluble protein could dramatically reduce SARS-CoV-2 infection in neuron cells, suggesting its critical function in mediating viral entry in neurons. Moreover, another confirmed ACE2-independent candidate receptor, CLEC4G, was known to be highly expressed in the liver (Domínguez-Soto et al., 2009), lymph node and monocytes (Figure S9B in Supporting Information). This gene encodes a glycan-binding receptor and is a member of the C-type lectin family, which has been found to facilitate SARS-CoV at- 
tachment through glycan-binding (Gramberg et al., 2005). Herein, we demonstrated for the first time CLEC4G's role in SARS-CoV-2 entry. Interestingly, the transmembrane protein TMEM30A was also identified in a recent genome-wide knockout screen for SARS-CoV-2 in Huh7.5 cells (Baggen et al., 2021). Our study confirmed its essentiality for SARS$\mathrm{CoV}-2$ entry and its direct binding with SARS-CoV-2 S. It is worth further investigation of the roles of LDLRAD3, CLEC4G, and TMEM30A in other tissue samples of COVID-19 patients where they are highly expressed. The discovery of multiple receptors in this study, with either tissuespecific or broad-spectrum expression patterns, might provide clues for understanding the multiorgan tropism of SARS-CoV-2 (Puelles et al., 2020).

It is worth noting that all these receptors we identified bind to the NTD domain of S, rather than RBD, the ACE2-binding domain, suggesting that NTD of SARS-CoV-2 S also plays important roles in mediating virus entry. Recent reports showed that NTD-specific neutralization antibodies isolated from convalescent COVID-19 patients enabled robust protection from the SARS-CoV-2 challenge (Chi et al., 2020; McCallum et al., 2021). It is possible that NTD-targeting antibodies might function by blocking NTD mediated virus entry.

Besides membrane proteins, we have also discovered several proteases, i.e., STAMBPL1 and TMPRSS15, whose overexpression promoted SARS-CoV-2 infection. The synergy of receptors and proteases in different tissues is worth of further investigation. Finally, the novel identified receptors or other functional factors brought a more comprehensive understanding for SARS-CoV-2 infection and might serve as novel therapeutic targets for COVID-19.

\section{MATERIALS AND METHODS}

\section{Plasmids}

The lentiviral sgRNA ${ }^{\mathrm{eBAR}}$-expressing backbone was constructed by inserting sgRNA scaffold embedded MS2 loops at tetraloop and stemloop 2 along with eBAR sequence into pLenti-sgRNA-Lib (Addgene, 53121). The sgRNA-expressing sequences were cloned into the backbone using the BsmBI-mediated Golden Gate cloning strategy (Engler et al., 2009). The pLenti-EGFP used for pseudovirus production was constructed by cloning EGFP sequence into pLentiSV40-mCherry. The cDNA-expressing plasmids were constructed by inserting each cDNA sequence into the multiple cloning sites before the Flag tag of the pLenti-SV40mCherry vector following the standard cloning protocol. The plasmids lenti dCAS-VP64_Blast (Addgene, 61425) and lenti MS2-P65-HSF1_Hygro (Addgene, 61426) were purchased from Addgene. The oligos of CRISPRa library were synthesized in Synbio Technologies according to the Human
Genome-wide CRISPRa-v2 Libraries (Addgene, 83978) (Horlbeck et al., 2016).

\section{Cell lines and culture conditions}

The HEK293T cell line was from EdiGene Inc., and Huh 7.5 cell line was from S. Cohen's laboratory (Stanford University School of Medicine). All these cells were maintained in Dulbecco's modified Eagle's medium (DMEM; Gibco, C11995500BT) supplemented with $10 \%$ fetal bovine serum (FBS; Biological Industries, 04-001-1ACS) and 1\% penicillin/streptomycin, and cultured with $5 \% \mathrm{CO}_{2}$ at $37^{\circ} \mathrm{C}$. Sf 21 insect cells were maintained in SIM SF medium (Sino Biological, RZ13NO0801) and $1 \%$ penicillin/streptomycin (Gibco, 2257215) with $110 \mathrm{r} \mathrm{min}^{-1}$ at $27^{\circ} \mathrm{C}$. All cells were routinely checked to confirm the absence of mycoplasma contamination.

\section{Production and infection of SARS-CoV-2 pseudotyped virus}

HEK293T cells were seeded $24 \mathrm{~h}$ before pseudovirus packaging. The SARS-CoV-2 pseudotyped virus was generated by co-transfection of the pCAGGS-S with the viral packaging plasmid psPAX2 and pLenti-EGFP/luciferaseexpressing plasmid as a proportion of 1:1:1 into HEK293T cells using the X-tremeGENE HP DNA transfection reagent (Roche, 06366546001) according to the manufacturer's instructions. The cell supernatant containing pseudovirus was collected $48 \mathrm{~h}$ post transfection, and was directly concentrated in different ratios using Lenti- $\mathrm{X}^{\mathrm{TM}}$ Concentrator (Clontech, 631232). The concentrated pseudovirus was immediately added into cells for infection without freezethawing. For infection with SARS-CoV-2 pseudotyped virus, cells were seeded $24 \mathrm{~h}$ before virus collecting. Concentrated pseudovirus was added into the culturing medium with polybrene $\left(8 \mu \mathrm{g} \mathrm{mL}^{-1}\right)$. After $24 \mathrm{~h}$, the medium was changed by conventional medium and cells were incubated for another $48 \mathrm{~h}$.

\section{Production of the SARS-CoV-2 authentic virus}

The SARS-CoV-2 authentic virus used in this study was isolated from the throat swab of a COVID-19 patient in Beijing and grown in Vero E6 cells in DMEM supplemented with $10 \%$ FBS. The TCID50 assay was used to determine the infectious titer of cultured SARS-CoV-2 virus.

\section{Construction of the CRISPRa sgRNA ${ }^{\mathrm{eBAR}}$ plasmid library}

The synthesized oligo pool of CRISPRa library was PCR amplified with primers (Table S5 in Supporting Information) 
including the BsmBI recognition sites using Phusion $₫$ HighFidelity PCR Kit (NEB, E0553L). After purification with DNA Clean \& Concentrator-25 (Zymo Research Corporation, D4034), the purified PCR product was respectively inserted into the three $\operatorname{sgRNA}^{\mathrm{eBAR}}$-expressing backbones constructed above through the Golden Gate cloning strategy (Engler et al., 2009). The ligation mixture of each group was separately purified with DNA Clean \& Concentrator-5 (Zymo Research Corporation, D4014), and was electrotransformed into E. coli HST08 Premium Electro-Cells (Takara, 9028) according to the manufacturer's protocol using a Gene Pulser Xcell (BioRad). Transformed clones were counted to ensure at least 300 -fold coverage for each sgRNA $^{\text {eBAR }}$. The plasmid of each sgRNA ${ }^{\text {eBAR }}$ library was extracted using an EndoFree Plasmid Maxi Kit (QIAGEN, 12362), and further mixed in a 1:1:1 molar ratio. The library lentivirus was generated by co-transfection of the library plasmid mixture with two lentiviral packaging plasmids pR8.74 and pVSV-G (Addgene, 12259) as a proportion of 10:10:1 into HEK293T cells. The cell supernatant containing lentivirus was collected $48 \mathrm{~h}$ post transfection and stored at $80^{\circ} \mathrm{C}$.

\section{CRISPRa screening for SARS-CoV-2 entry factors}

The HEK293T cells were engineered to stably express the CRISPRa system including lenti dCAS-VP64_Blast and lenti MS2-P65-HSF1_Hygro vectors, termed as HEK293TCRISPRa cells. The HEK293T-CRISPRa cells were seeded $24 \mathrm{~h}$ post lentiviral infection, and were further infected with the library lentivirus at an MOI of 10 with a high coverage (5,000-fold) for each sgRNA. Two days post lentiviral infection, the library cells were subjected to puromycin selection for $48 \mathrm{~h}\left(1 \mu \mathrm{g} \mathrm{mL}^{-1}\right)$. After puromycin treatment, the library cells were collected as the reference sample and were continuously cultured for 5 days. The fresh SARS-CoV-2 pseudovirus with EGFP marker (50-fold) was added to the library cells, and the EGFP-positive cells were sorted by FACS $48 \mathrm{~h}$ post first round of pseudovirus infection. After culturing the sorted cells for additional several days, a second round of pseudovirus infection was conducted as described above, and the library cells were sorted for total $\mathrm{EGFP}^{+}$cells as well as the top $10 \%-20 \%$, top $10 \%$ and top $2 \%$ grouped by the EGFP intensity. The reference sample and each group of $\mathrm{EGFP}^{+}$cells were subjected to genomic extraction (QIAGEN, 69506), PCR amplification of the sgRNA $^{\text {eBAR }}$ sequences (KAPA, KK2631) and high-throughput sequencing as previously described (Liu et al., 2018).

\section{Analysis of CRISPRa screening results}

In order to calculate the enriched genes after CRISPRa screening, we developed an analysis algorithm eBAR-ana- lyzer, which was implemented using $\mathrm{R}$ and could be obtained from https://github.com/wolfsonliu/FluorescenceSelection. In principle, the eBAR-analyzer algorithm adopted binomial distribution, in which the selection of cells hosting sgRNAs targeting specific genes enriched by FACS was considered as results of a series of Bernoulli trials. The normalization of raw counts of sgRNAs ${ }^{\text {eBAR }}$ was calculated based on the cell proportion of EGFP intensity groups compared with the initial cell population. For instance, when we selected the top $2 \%$ intensity of $\mathrm{EGFP}^{+}$cells, the normalization factor for the group will be 0.02 . Then the normalized counts would be total detected reads multiplied by group normalization factor. The final normalization process would ensure that the smallest normalized counts will be an integer after rounded. Based on this, the $P$-values of the sgRNAs ${ }^{\text {eBAR }}$ were calculated by assuming counts of each intensity group were drawn from the initial population counts satisfying a binomial distribution. The normalized ranks of $P$-values for each sgRNA ${ }^{\text {eBAR }}$ were calculated. Finally, Robust Rank Aggregation (RRA) (Kolde et al., 2012) was used to calculate the rank in gene level from the normalized ranks of $P$-values of $\operatorname{sgRNAs}{ }^{\text {eBAR }}$. The RRA scores were the output results of the algorithm.

\section{GO enrichment and expression pattern analysis}

The Gene Ontology (GO) enrichment analysis of identified host factors (RRA score $<0.001$ ) was performed using Metascape Resource (Zhou et al., 2019). Hypergeometric test was used to calculate all the $P$-values for all the terms. We selected top-enriched GO terms for visualization in this manuscript. For the expression pattern analysis of identified candidates, we used data retrieved from Human Protein Atlas to obtain normalized expression of each factor (Uhlén et al., 2015).

\section{Validation of identified candidates}

For the individual validation of screening results, we introduced cDNAs of candidate genes into cells. The cDNAs were transfected into cells using the X-tremeGENE HP DNA transfection reagent (Roche, 06366546001). Then the transfected cells were infected by concentrated SARS-CoV-2 pseudotyped virus $48 \mathrm{~h}$ later. The infection of pseudotyped virus was quantified through measuring luciferase activities.

The lentiviral particles expressing individual cDNA labelled with an mCherry marker were generated by cotransfection of the cDNA plasmid mixture with two lentiviral packaging plasmids pR8.74 and pVSV-G (Addgene, 12259) as a proportion of 10:10:1 into HEK293 cells, followed by infection into cells. The cDNA transduced cells were selected through FACS and were infected with authentic SARS-CoV-2 virus at an MOI of 0.5 for $1 \mathrm{~h}$. Infected cells 
were cultured for another $24 \mathrm{~h}$ with conventional medium, then treated with Trizol. The infection of authentic SARSCoV-2 virus was quantified by real-time qPCR of RNA abundance.

\section{Protein production and purification}

The SARS-CoV-2 NTD (residues 13-303) or RBD (residues 319-541) with a C-terminal His tag was cloned into a modified pFastBac vector (Invitrogen) that encodes a melittin signal peptide before the NTD or RBD. Bacmids DNA were generated using the Bac-to-Bac system. Baculoviruses were generated and amplified using the $\mathrm{Sf} 21$ insect cells, and were subsequently used to infect High Five insect cells for protein expression. NTD/RBD was retrieved from the conditioned cell growth media using the Ni-NTA resin and further purified using a Superdex 200 Increase 10/300 gel filtration column in $20 \mathrm{mmol} \mathrm{L}^{-1}$ HEPES $\mathrm{pH} 7.2$, and $150 \mathrm{mmol} \mathrm{L}^{-1} \mathrm{NaCl}$. Strep-tagged S6P spike protein was expressed in the HEK293F cells and purified as described previously (Du et al., 2020).

\section{Co-IP}

For the Co-IP assay, the plasmids of SARS-CoV-2 S6P spike and individual cDNA were transfected into HEK293T cells. After $48 \mathrm{~h}$, the cells were washed using precooled PBS for three times, then lysed with precooled lysis buffer (50 $\mathrm{mmol} \mathrm{L}^{-1}$ Tris- $\mathrm{HCl} \mathrm{pH} 7.4,150 \mathrm{mmol} \mathrm{L}^{-1} \mathrm{NaCl}, 0.25 \%$ Na-deoxycholate, $1 \mathrm{mmol} \mathrm{L}^{-1}$ EDTA, $1 \%$ NonidetP-40) with Protease Inhibitor Cocktail Tablet (Thermo, VJ313124) at $4^{\circ} \mathrm{C}$ for $1 \mathrm{~h}$ before being subjected to centrifugation at $15,000 \times g$ at $4^{\circ} \mathrm{C}$ for $15 \mathrm{~min}$. We transferred $30 \mu \mathrm{L}$ sample into a new tube as the input. The rest of cell lysates were treated with Anti-Flag M2 Affinity Gel (Sigma, A2220) at $4^{\circ}$ $\mathrm{C}$ overnight. Then the lysates were washed using wash buffer (50 mmol L ${ }^{-1}$ Tris- $\mathrm{HCl} \mathrm{pH} 7.4,150 \mathrm{mmol} \mathrm{L}^{-1} \mathrm{NaCl}, 0.25 \%$ Na-deoxycholate, $1 \mathrm{mmol} \mathrm{L}^{-1}$ EDTA, $0.1 \%$ NonidetP-40) for at least four times. The proteins were eluted using $250 \mu \mathrm{g} \mathrm{mL} L^{-1}$ Flag peptide into wash buffer for $1 \mathrm{~h}$ at $4{ }^{\circ} \mathrm{C}$ and subjected to immunoblotting analysis using antibodies for Flag tag (SIGMA, SLCD6338) and SARS-CoV-2 S (Sino Biological, 40589-T62).

\section{Flag pull-down assay}

Potential receptor proteins with C-terminal Flag tag were transiently expressed in HEK293F cells using polyethylenimine (PEI, Polysciences). Thirty-six hours following transfection, the cells were collected by centrifugation and disrupted on ice using a dounce homogenizer in the lysis buffer $\left(25 \mathrm{mmol} \mathrm{L}^{-1}\right.$ Tris pH 8.0, $150 \mathrm{mmol} \mathrm{L}^{-1} \mathrm{NaCl}, 1.0 \%$ $(\mathrm{w} / \mathrm{v}) \mathrm{N}$-dodecyl $\beta$-d-maltoside (DDM), and $0.1 \%(\mathrm{w} / \mathrm{v})$ cholesterol hemisuccinate (CHS)), supplemented with Protease Inhibitor cocktail (Bimake, B14001). After ultracentrifugation $\left(45,000 \times \mathrm{g}, 30 \mathrm{~min}, 4^{\circ} \mathrm{C}\right)$, the supernatants were first incubated with the anti-Flag affinity beads (SmartLifesciences, SA042025) for $2 \mathrm{~h}$ at $4^{\circ} \mathrm{C}$ with rotation. The beads were then pelleted and washed for five times with the wash buffer $\left(25 \mathrm{mmol} \mathrm{L}^{-1}\right.$ Tris pH 8.0, $150 \mathrm{mmol} \mathrm{L}^{-1} \mathrm{NaCl}$, $0.3 \%(\mathrm{w} / \mathrm{v}) \mathrm{DDM}$, and $0.03 \%(\mathrm{w} / \mathrm{v}) \mathrm{CHS})$. Afterwards, the beads were incubated with purified S6P/NTD/RBD proteins as described above for $1 \mathrm{~h}$ with rotation. The beads were then again pelleted and washed five times with the wash buffer. Bound proteins were eluted from the beads using the elute buffer $\left(25 \mathrm{mmol} \mathrm{L}^{-1}\right.$ Tris pH 8.0, $150 \mathrm{mmol} \mathrm{L}^{-1} \mathrm{NaCl}, 0.1 \%$ (w/v) DDM, 0.01\% (w/v) CHS, and $250 \mathrm{ng} \mathrm{mL}^{-1}$ Flag peptide), and analysed by immunoblotting using antibodies for the Strep tag (HuaxingBio, HX1816) or His tag (TransGen, HT501).

\section{StrepTactin pull-down assay}

For the StrepTactin pull-down assay, potential receptor proteins were purified using the anti-Flag affinity beads and eluted as described above. Then they were incubated with purified S6P on ice for $1 \mathrm{~h}$. The mixtures were then incubated with the StrepTactin beads (Smart Lifesciences) in the wash buffer at $4^{\circ} \mathrm{C}$ for another $1 \mathrm{~h}$ with rotation, washed five times with the wash buffer, and eluted using the final buffer $\left(25 \mathrm{mmol} \mathrm{L}^{-1}\right.$ Tris $\mathrm{pH} 8.0,150 \mathrm{mmol} \mathrm{L}^{-1} \mathrm{NaCl}, 0.1 \%$ (w/v) DDM, $0.01 \%(\mathrm{w} / \mathrm{v}) \mathrm{CHS}$, and $10 \mathrm{mmol} \mathrm{L}^{-1}$ desthiobiotin). The results were analysed by immunoblotting using antibodies for the Flag tag (SIGMA, SLCD6338).

\section{SPR measurements}

The dynamic interactions between candidate receptors and SARS-CoV-2 S were measured using Biacore T200 system (GE Healthcare). Purified SARS-CoV-2 S protein was captured with $400 \mathrm{RU}$ on a CM5 sensor chip (GE Healthcare) according to the manufacturer's instructions. The chip surface was regenerated by $10 \mathrm{mmol} \mathrm{L}^{-1}$ glycine- $\mathrm{HCl} \mathrm{pH} \mathrm{1.5.} \mathrm{All} \mathrm{the}$ measurements were performed in the buffer containing $10 \mathrm{mmol} \mathrm{L}^{-1}$ HEPES $\mathrm{pH} \quad 7.4, \quad 0.05 \%$ Tween-20 and $150 \mathrm{mmol} \mathrm{L}^{-1} \mathrm{NaCl}$ with a flow rate of $30 \mu \mathrm{L} \mathrm{min}^{-1}$ at $25^{\circ} \mathrm{C}$. Different concentrations of candidate receptors $(0$, $0.03125 \mu \mathrm{mol} \mathrm{L}^{-1}, \quad 0.0625 \mu \mathrm{mol} \mathrm{L}{ }^{-1}, \quad 0.125 \mu \mathrm{mol} \mathrm{L}{ }^{-1}$, $0.25 \mu \mathrm{mol} \mathrm{L}{ }^{-1}, 0.5 \mu \mathrm{mol} \mathrm{L}{ }^{-1}$ and $1 \mu \mathrm{mol} \mathrm{L}^{-1}$ ) were injected for $180 \mathrm{~s}$ per cycle with the monitored dissociation of $600 \mathrm{~s}$. Kinetic association and dissociation curves at each concentration were calculated using the Biacore evaluation software.

\section{Syncytium formation assay}

Wild-type HEK293T cells or HEK293T $A C E 2^{-/-}$cells were 
first transfected with the pCAGGS-S plasmid with an EGFP selection marker. Twenty-four hours post transfection, the transfected cells were detached, and mixed with HEK293T cells stably overexpressing different cDNAs (ACE2, CLEC4G, LDLRAD3, TMEM30A and the cDNA-expressing empty vector as the negative control) labelled with an mCherry marker in a 1:1 ratio. Then the two categories of cells were co-cultured in 12 -well plates at about $60 \%$ confluency. After $40 \mathrm{~h}$ of cell co-culture, the images were captured by fluorescence microscopy.

\section{Real-time qPCR}

The cultured cells transfected with siRNAs or/and infected with authentic SARS-CoV-2 virus were treated by Trizol. RNA was extracted using Direct-zol RNA kit (Zymo, R2069), and the cDNA was synthesized using QuantScript RT kit (TIANGEN, KR103-03). Real-time PCR was performed using SYBR Premix Ex Taq II (TaKaRa, RR820A) on LightCycler96 qPCR system (Roche). The relative RNA abundance of candidate factors or SARS-CoV-2 virus was measured and normalized by GAPDH. All the primers used for real-time qPCR are listed in Table S5 in Supporting Information.

\section{Inhibition of SARS-CoV-2 infection by soluble proteins and siRNAs}

For inhibition by soluble proteins, the purified protein of ACE2, LDLRAD3 or CLEC4G with different doses (0, $12.5 \mu \mathrm{g} \mathrm{mL}^{-1}, 25 \mu \mathrm{g} \mathrm{mL}^{-1}, 50 \mu \mathrm{g} \mathrm{mL}^{-1}$ and $100 \mu \mathrm{g} \mathrm{mL}^{-1}$ ) were incubated with authentic SARS-CoV-2 virus for $1 \mathrm{~h}$ followed by infection at an MOI of 0.5 . For inhibition by siRNAs, cells were seeded at 24-well plates $24 \mathrm{~h}$ before transfection. Each siRNA including negative control siRNA at an amount of 20 pmol was transfected into cells with $6 \mu \mathrm{L}$ Lipofectamine RNAiMAX Reagent (Life technologies, 13778-150). Twenty-four hours later, the cells were infected with authentic SARS-CoV-2 virus at an MOI of 0.5 for $1 \mathrm{~h}$. Infected cells were cultured for another $24 \mathrm{~h}$ with conventional medium, then treated with Trizol. The infection of authentic SARS-CoV-2 virus was quantified by real-time qPCR of RNA abundance.

\section{Statistical analyses}

Statistical analysis of all data apart from CRISPRa screening was performed using GraphPad Prism software. The statistical significance was evaluated using Student's $t$ test and determined as $P<0.05$. $P$-values were indicated in each of figure legends.

Compliance and ethics The author(s) declare that they have no conflict of interest.

Acknowledgements We acknowledge the National Center for Protein Sciences (Beijing) at Peking University for their assistance with fluorescence-activated cell sorting and SPR measurements, particularly Dr. Jia Luo, Ms Huan Yang, Ms Liying Du and Ms Hui Li for their technical help. We acknowledge Dr. Ying Yu (Peking University) for her assistance in preparing the NGS library. This project was supported by funds from the National Key R\&D Program of China (2020YFA0707800 to W.W., 2020 YFA0707600 to Z.Z.), Beijing Municipal Science \& Technology Commission (Z181100001318009), the National Natural Science Foundation of China (31930016), Beijing Advanced Innovation Center for Genomics at Peking University and the Peking-Tsinghua Center for Life Sciences (to W. $W$.), the National Natural Science Foundation of China (31870893), the National Major Science \& Technology Project for Control and Prevention of Major Infectious Diseases in China (2018ZX10301401 to Z.Z.), and China Postdoctoral Science Foundation (2020M670031 to Y.L.).

\section{References}

Aguiar, J.A., Tremblay, B.J.M., Mansfield, M.J., Woody, O., Lobb, B., Banerjee, A., Chandiramohan, A., Tiessen, N., Cao, Q., DvorkinGheva, A., et al. (2020). Gene expression and in situ protein profiling of candidate SARS-CoV-2 receptors in human airway epithelial cells and lung tissue. Eur Respir J 56, 2001123.

Al-Sharaky, D.R., Kandil, M.A.E.H., Aiad, H.A.S., El-Hosary, E.M., Alagizy, H.A., Elshenawy, M.A.S., and El-Rebey, H.S. (2020). ROC-1, P21 and CAIX as markers of tumor aggressiveness in bladder carcinoma in Egyptian patients. Diagn Pathol 15, 33.

Andersen, K.G., Rambaut, A., Lipkin, W.I., Holmes, E.C., and Garry, R.F. (2020). The proximal origin of SARS-CoV-2. Nat Med 26, 450-452.

Baggen, J., Persoons, L., Vanstreels, E., Jansen, S., Van Looveren, D., Boeckx, B., Geudens, V., De Man, J., Jochmans, D., Wauters, J., et al. (2021). Genome-wide CRISPR screening identifies TMEM106B as a proviral host factor for SARS-CoV-2. Nat Genet 53, 435-444.

Buchrieser, J., Dufloo, J., Hubert, M., Monel, B., Planas, D., Rajah, M.M., Planchais, C., Porrot, F., Guivel-Benhassine, F., Van der Werf, S., et al. (2021). Syncytia formation by SARS-CoV-2-infected cells. EMBO J 40, e107405.

Cantuti-Castelvetri, L., Ojha, R., Pedro, L.D., Djannatian, M., Franz, J., Kuivanen, S., van der Meer, F., Kallio, K., Kaya, T., Anastasina, M., et al. (2020). Neuropilin-1 facilitates SARS-CoV-2 cell entry and infectivity. Science 370, 856-860.

Cheung, K.S., Hung, I.F.N., Chan, P.P.Y., Lung, K.C., Tso, E., Liu, R., Ng, Y.Y., Chu, M.Y., Chung, T.W.H., Tam, A.R., et al. (2020). Gastrointestinal manifestations of SARS-CoV-2 infection and virus load in fecal samples from a Hong Kong cohort: systematic review and meta-analysis. Gastroenterology 159, 81-95.

Chi, X., Yan, R., Zhang, J., Zhang, G., Zhang, Y., Hao, M., Zhang, Z., Fan, P., Dong, Y., Yang, Y., et al. (2020). A neutralizing human antibody binds to the N-terminal domain of the Spike protein of SARS-CoV-2. Science 369, 650-655.

Daly, J.L., Simonetti, B., Klein, K., Chen, K.E., Williamson, M.K., AntónPlágaro, C., Shoemark, D.K., Simón-Gracia, L., Bauer, M., Hollandi, R., et al. (2020). Neuropilin-1 is a host factor for SARS-CoV-2 infection. Science 370, 861-865.

Daniloski, Z., Jordan, T.X., Wessels, H.H., Hoagland, D.A., Kasela, S., Legut, M., Maniatis, S., Mimitou, E.P., Lu, L., Geller, E., et al. (2021). Identification of required host factors for SARS-CoV-2 infection in human cells. Cell 184, 92-105.e16.

Dieterle, M.E., Haslwanter, D., Bortz Iii, R.H., Wirchnianski, A.S., Lasso, G., Vergnolle, O., Abbasi, S.A., Fels, J.M., Laudermilch, E., Florez, C., et al. (2020). A replication-competent vesicular stomatitis virus for studies of SARS-CoV-2 spike-mediated cell entry and its inhibition. Cell Host Microbe 28, 486-496.e6.

Domínguez-Soto, A., Aragoneses-Fenoll, L., Gómez-Aguado, F., Corcuera, 
M.T., Clária, J., García-Monzón, C., Bustos, M., and Corbí, A.L. (2009). The pathogen receptor liver and lymph node sinusoidal endotelial cell C-type lectin is expressed in human Kupffer cells and regulated by PU.1. Hepatology 49, 287-296.

Du, S., Cao, Y., Zhu, Q., Yu, P., Qi, F., Wang, G., Du, X., Bao, L., Deng, W., Zhu, H., et al. (2020). Structurally resolved SARS-CoV-2 antibody shows high efficacy in severely infected hamsters and provides a potent cocktail pairing strategy. Cell 183, 1013-1023.e13.

Engler, C., Gruetzner, R., Kandzia, R., and Marillonnet, S. (2009). Golden gate shuffling: a one-pot DNA shuffling method based on type IIs restriction enzymes. PLoS ONE 4, e5553.

Fan, M., Wang, J., Wang, S., Li, T., Pan, H., Liu, H., Xu, H., Zhernakova, D.V., O'Brien, S.J., Feng, Z., et al. (2020). New gene variants associated with the risk of chronic HBV infection. Virol Sin 35, 378-387.

Gramberg, T., Hofmann, H., Möller, P., Lalor, P.F., Marzi, A., Geier, M., Krumbiegel, M., Winkler, T., Kirchhoff, F., Adams, D.H., et al. (2005). LSECtin interacts with filovirus glycoproteins and the spike protein of SARS coronavirus. Virology 340, 224-236.

Grant, R.A., Morales-Nebreda, L., Markov, N.S., Swaminathan, S., Querrey, M., Guzman, E.R., Abbott, D.A., Donnelly, H.K., Donayre, A., Goldberg, I.A., et al. (2021). Circuits between infected macrophages and T cells in SARS-CoV-2 pneumonia. Nature 590, 635-641.

Helms, J., Kremer, S., Merdji, H., Clere-Jehl, R., Schenck, M., Kummerlen, C., Collange, O., Boulay, C., Fafi-Kremer, S., Ohana, M., et al. (2020). Neurologic features in severe SARS-CoV-2 infection. N Engl J Med $382,2268-2270$.

Hiraizumi, M., Yamashita, K., Nishizawa, T., and Nureki, O. (2019). CryoEM structures capture the transport cycle of the P4-ATPase flippase. Science 365, 1149-1155.

Hoffmann, M., Kleine-Weber, H., and Pöhlmann, S. (2020a). A multibasic cleavage site in the spike protein of SARS-CoV-2 is essential for infection of human lung cells. Mol Cell 78, 779-784.e5.

Hoffmann, M., Kleine-Weber, H., Schroeder, S., Krüger, N., Herrler, T., Erichsen, S., Schiergens, T.S., Herrler, G., Wu, N.H., Nitsche, A., et al. (2020b). SARS-CoV-2 cell entry depends on ACE2 and TMPRSS2 and is blocked by a clinically proven protease inhibitor. Cell 181, 271-280. e8.

Horlbeck, M.A., Gilbert, L.A., Villalta, J.E., Adamson, B., Pak, R.A., Chen, Y., Fields, A.P., Park, C.Y., Corn, J.E., Kampmann, M., et al. (2016). Compact and highly active next-generation libraries for CRISPR-mediated gene repression and activation. eLife 5, e19760.

Huang, C., Wang, Y., Li, X., Ren, L., Zhao, J., Hu, Y., Zhang, L., Fan, G., $\mathrm{Xu}, \mathrm{J}$., Gu, X., et al. (2020). Clinical features of patients infected with 2019 novel coronavirus in Wuhan, China. Lancet 395, 497-506.

Karaca, E., Harel, T., Pehlivan, D., Jhangiani, S.N., Gambin, T., Coban Akdemir, Z., Gonzaga-Jauregui, C., Erdin, S., Bayram, Y., Campbell, I. M., et al. (2015). Genes that affect brain structure and function identified by rare variant analyses of Mendelian neurologic disease. Neuron 88, 499-513.

Kolde, R., Laur, S., Adler, P., and Vilo, J. (2012). Robust rank aggregation for gene list integration and meta-analysis. Bioinformatics 28, 573-580.

Konermann, S., Brigham, M.D., Trevino, A.E., Joung, J., Abudayyeh, O. O., Barcena, C., Hsu, P.D., Habib, N., Gootenberg, J.S., Nishimasu, H., et al. (2015). Genome-scale transcriptional activation by an engineered CRISPR-Cas9 complex. Nature 517, 583-588.

Lan, J., Ge, J., Yu, J., Shan, S., Zhou, H., Fan, S., Zhang, Q., Shi, X., Wang, Q., Zhang, L., et al. (2020). Structure of the SARS-CoV-2 spike receptor-binding domain bound to the ACE2 receptor. Nature 581, 215220.

Liu, Y., Cao, Z., Wang, Y., Guo, Y., Xu, P., Yuan, P., Liu, Z., He, Y., and Wei, W. (2018). Genome-wide screening for functional long noncoding RNAs in human cells by Cas9 targeting of splice sites. Nat Biotechnol 36, 1203-1210.

Lukassen, S., Chua, R.L., Trefzer, T., Kahn, N.C., Schneider, M.A., Muley, T., Winter, H., Meister, M., Veith, C., Boots, A.W., et al. (2020). SARS$\mathrm{CoV}-2$ receptor ACE2 and TMPRSS2 are primarily expressed in bronchial transient secretory cells. EMBO J 39, e105114.
Ma, H., Kim, A.S., Kafai, N.M., Earnest, J.T., Shah, A.P., Case, J.B., Basore, K., Gilliland, T.C., Sun, C., Nelson, C.A., et al. (2020). LDLRAD3 is a receptor for Venezuelan equine encephalitis virus. Nature 588, 308-314.

Madjid, M., Safavi-Naeini, P., Solomon, S.D., and Vardeny, O. (2020). Potential effects of coronaviruses on the cardiovascular system. JAMA Cardiol 5, 831-840.

Matsuyama, S., Nao, N., Shirato, K., Kawase, M., Saito, S., Takayama, I., Nagata, N., Sekizuka, T., Katoh, H., Kato, F., et al. (2020). Enhanced isolation of SARS-CoV-2 by TMPRSS2-expressing cells. Proc Natl Acad Sci USA 117, 7001-7003.

McCallum, M., De Marco, A., Lempp, F.A., Tortorici, M.A., Pinto, D., Walls, A.C., Beltramello, M., Chen, A., Liu, Z., Zatta, F., et al. (2021). $\mathrm{N}$-terminal domain antigenic mapping reveals a site of vulnerability for SARS-CoV-2. Cell 184, 2332-2347.e16.

Ou, X., Liu, Y., Lei, X., Li, P., Mi, D., Ren, L., Guo, L., Guo, R., Chen, T., $\mathrm{Hu}$, J., et al. (2020). Characterization of spike glycoprotein of SARS$\mathrm{CoV}-2$ on virus entry and its immune cross-reactivity with SARS-CoV. Nat Commun 11, 1620.

Puelles, V.G., Lütgehetmann, M., Lindenmeyer, M.T., Sperhake, J.P., Wong, M.N., Allweiss, L., Chilla, S., Heinemann, A., Wanner, N., Liu, S., et al. (2020). Multiorgan and renal tropism of SARS-CoV-2. N Engl J Med 383, 590-592.

Puray-Chavez, M., LaPak, K.M., Schrank, T.P., Elliott, J.L., Bhatt, D.P., Agajanian, M.J., Jasuja, R., Lawson, D.Q., Davis, K., Rothlauf, P.W., et al. (2021). Systematic analysis of SARS-CoV-2 infection of an ACE2negative human airway cell. Cell Rep 36, 109364.

Ranganathan, S., Noyes, N.C., Migliorini, M., Winkles, J.A., Battey, F.D., Hyman, B.T., Smith, E., Yepes, M., Mikhailenko, I., and Strickland, D. K. (2011). LRAD3, a novel low-density lipoprotein receptor family member that modulates amyloid precursor protein trafficking. J Neurosci 31, 10836-10846.

Ren, L.L., Wang, Y.M., Wu, Z.Q., Xiang, Z.C., Guo, L., Xu, T., Jiang, Y.Z., Xiong, Y., Li, Y.J., Li, X.W., et al. (2020). Identification of a novel coronavirus causing severe pneumonia in human: a descriptive study. Chin Med J 133, 1015-1024.

Ren, X., Wen, W., Fan, X., Hou, W., Su, B., Cai, P., Li, J., Liu, Y., Tang, F., Zhang, F., et al. (2021). COVID-19 immune features revealed by a large-scale single-cell transcriptome atlas. Cell 184, 1895-1913.e19.

Shang, J., Ye, G., Shi, K., Wan, Y., Luo, C., Aihara, H., Geng, Q., Auerbach, A., and Li, F. (2020). Structural basis of receptor recognition by SARS-CoV-2. Nature 581, 221-224.

Simmons, G., Zmora, P., Gierer, S., Heurich, A., and Pöhlmann, S. (2013). Proteolytic activation of the SARS-coronavirus spike protein: cutting enzymes at the cutting edge of antiviral research. Antiviral Res 100, 605-614.

Trump, S., Lukassen, S., Anker, M.S., Chua, R.L., Liebig, J., Thürmann, L., Corman, V.M., Binder, M., Loske, J., Klasa, C., et al. (2021). Hypertension delays viral clearance and exacerbates airway hyperinflammation in patients with COVID-19. Nat Biotechnol 39, 705-716.

Uhlén, M., Fagerberg, L., Hallström, B.M., Lindskog, C., Oksvold, P., Mardinoglu, A., Sivertsson, Å., Kampf, C., Sjöstedt, E., Asplund, A., et al. (2015). Tissue-based map of the human proteome. Science 347, 1260419.

Walls, A.C., Park, Y.J., Tortorici, M.A., Wall, A., McGuire, A.T., and Veesler, D. (2020). Structure, function, and antigenicity of the SARSCoV-2 spike glycoprotein. Cell 181, 281-292.e6.

Wang, D., Hu, B., Hu, C., Zhu, F., Liu, X., Zhang, J., Wang, B., Xiang, H., Cheng, Z., Xiong, Y., et al. (2020). Clinical characteristics of 138 hospitalized patients with 2019 novel coronavirus-infected pneumonia in Wuhan, China. JAMA 323, 1061-1069.

Wang, S., Qiu, Z., Hou, Y., Deng, X., Xu, W., Zheng, T., Wu, P., Xie, S., Bian, W., Zhang, C., et al. (2021). AXL is a candidate receptor for SARS-CoV-2 that promotes infection of pulmonary and bronchial epithelial cells. Cell Res 31, 126-140.

Wei, J., Alfajaro, M.M., DeWeirdt, P.C., Hanna, R.E., Lu-Culligan, W.J., 
Cai, W.L., Strine, M.S., Zhang, S.M., Graziano, V.R., Schmitz, C.O., et al. (2021). Genome-wide CRISPR screens reveal host factors critical for SARS-CoV-2 infection. Cell 184, 76-91.e13.

Wrapp, D., Wang, N., Corbett, K.S., Goldsmith, J.A., Hsieh, C.L., Abiona, O., Graham, B.S., and McLellan, J.S. (2020). Cryo-EM structure of the 2019-nCoV spike in the prefusion conformation. Science 367, 12601263.

Zhou, P., Yang, X.L., Wang, X.G., Hu, B., Zhang, L., Zhang, W., Si, H.R., Zhu, Y., Li, B., Huang, C.L., et al. (2020). A pneumonia outbreak associated with a new coronavirus of probable bat origin. Nature 579, 270-273.

Zhou, Y., Zhou, B., Pache, L., Chang, M., Khodabakhshi, A.H.,
Tanaseichuk, O., Benner, C., and Chanda, S.K. (2019). Metascape provides a biologist-oriented resource for the analysis of systems-level datasets. Nat Commun 10, 1523.

Zhu, N., Zhang, D., Wang, W., Li, X., Yang, B., Song, J., Zhao, X., Huang, B., Shi, W., Lu, R., et al. (2020). A novel coronavirus from patients with pneumonia in China, 2019. N Engl J Med 382, 727-733.

Zhu, S., Cao, Z., Liu, Z., He, Y., Wang, Y., Yuan, P., Li, W., Tian, F., Bao, Y., and Wei, W. (2019). Guide RNAs with embedded barcodes boost CRISPR-pooled screens. Genome Biol 20, 20.

Zhu, Y., Feng, F., Hu, G., Wang, Y., Yu, Y., Zhu, Y., Xu, W., Cai, X., Sun, Z., Han, W., et al. (2021). A genome-wide CRISPR screen identifies host factors that regulate SARS-CoV-2 entry. Nat Commun 12, 961.

\section{SUPPORTING INFORMATION}

The supporting information is available online at https://doi.org/10.1007/s11427-021-1990-5. The supporting materials are published as submitted, without typesetting or editing. The responsibility for scientific accuracy and content remains entirely with the authors. 